\title{
Hybrid method to resolve the neutrino mass hierarchy by supernova (anti)neutrino induced reactions
}

\author{
D. Vale ${ }^{1}$. T. Rauscher ${ }^{2,3}$, and N. Paar 3 , 讯 \\ ${ }^{1}$ Department of Physics, Faculty of Science, University of Zagreb, Croatia \\ ${ }^{2}$ Centre for Astrophysics Research, University of Hertfordshire, \\ College Lane, Hatfield AL10 9AB, United Kingdom and \\ ${ }^{3}$ Department of Physics, University of Basel, Klingelbergstrasse 82, CH-4056 Basel, Switzerland
}

(Dated: September 25, 2015)

\begin{abstract}
We introduce a hybrid method to determine the neutrino mass hierarchy by simultaneous measurements of responses of at least two detectors to antineutrino and neutrino fluxes from accretion and cooling phases of core-collapse supernovae. The (anti)neutrino-nucleus cross sections for ${ }^{56} \mathrm{Fe}$ and ${ }^{208} \mathrm{~Pb}$ are calculated in the framework of the relativistic nuclear energy density functional and weak interaction Hamiltonian, while the cross sections for inelastic scattering on free protons $\mathrm{p}\left(\bar{\nu}_{\mathrm{e}}, \mathrm{e}^{+}\right) \mathrm{n}$ are obtained using heavy-baryon chiral perturbation theory. The modelling of (anti)neutrino fluxes emitted from a protoneutron star in a core-collapse supernova include collective and Mikheyev-Smirnov-Wolfenstein effects inside the exploding star. The particle emission rates from the elementary decay modes of the daughter nuclei are calculated for normal and inverted neutrino mass hierarchy. It is shown that simultaneous use of (anti)neutrino detectors with different target material allows to determine the neutrino mass hierarchy from the ratios of $\nu_{\mathrm{e}}-$ and $\bar{\nu}_{\mathrm{e}}$-induced particle emissions. This hybrid method favors neutrinos from the supernova cooling phase and the implementation of detectors with heavier target nuclei $\left({ }^{208} \mathrm{~Pb}\right)$ for the neutrino sector, while for antineutrinos the use of free protons in mineral oil or water is the appropriate choice.
\end{abstract}

PACS numbers: 21.10.Gv,21.30.Fe,21.60.Jz,24.30.Cz

\section{INTRODUCTION}

Over the past years considerable progress has been achieved in constraining the mixing parameters in the neutrino oscillation framework [1, 2], based on various experiments involving atmospheric, solar, and terrestrial neutrinos [3]. It is now well established that neutrinos have non-vanishing rest masses and that the flavor states $\nu_{e}, \nu_{\mu}$, and $\nu_{\tau}$ are quantum mechanical mixtures of the vacuum mass eigenstates $\nu_{1}, \nu_{2}$, and $\nu_{3}$ [4]. However, currently existing data do not determine the neutrino mass hierarchy, i.e., the sign of mass squared difference $\Delta m_{31}^{2}=m_{3}^{2}-m_{1}^{2}$. In the case of $\Delta m_{31}^{2}>0$ one refers to the normal mass hierarchy ( $\mathrm{NH}$ ), while $\Delta m_{31}^{2}<0$ corresponds to the inverted mass hierarchy $(\mathrm{IH})$. Although a number of techniques has been proposed to resolve the neutrino mass hierarchy, to date this question still remains open and presents an important scientific challenge. Recent approaches to resolve the neutrino mass hierarchy include methods based on reactor neutrinos [5 7], various baseline experiments 8], Earth matter effects on the supernova neutrino signal [9, 10], the spectral swapping of supernova neutrino flavors [11], the rise time of supernova $\bar{\nu}_{\mathrm{e}}$ light curves [12], the analysis of meteoritic supernova material [13], and detection of atmospheric neutrinos in sea water or ice [14].

The neutrino burst from a core collapsing supernova (ccSN) provides valuable information not only on the explosion mechanism, but also about the neutrino mass hierarchy that is extremely difficult to determine in the laboratory [15]. The first observation of the neutrino burst, from supernova SN1987A in the Large Magellanic Cloud, paved the way for neutrino astronomy, and new frontiers in neutrino physics and astrophysics research. 16 18. Several supernovaneutrino detectors are currently running and a variety of new detectors are proposed to observe galactic ccSN burst 19 , 20]. In the anticipation of the next galactic supernova (the occurrence rate is $\approx 1-3$ events per century [15]), with expected high statistics of (anti)neutrino induced detector events, in this paper we introduce a hybrid method to resolve the neutrino mass hierarchy, based on neutrino and antineutrino reactions with ${ }^{56} \mathrm{Fe},{ }^{208} \mathrm{~Pb}$, and with free protons in terrestrial neutrino detectors. The aim is to explore novel perspectives for the implementation of $\nu_{\mathrm{e}}$ and $\bar{\nu}_{\mathrm{e}}$ detectors, based on various nuclei and/or protons (in mineral oil and water) as main target material. Since for supernova SN1987A mainly the $\bar{\nu}_{\mathrm{e}}$ signal has been detected, the role of neutrino induced detector events for understanding the fundamental properties of neutrinos remain largely unknown. While most of the supernova detectors based on

*Electronic address: dvale@phy.hr

$\dagger$ Electronic address: npaar@phy.hr 
free protons or nuclear targets still are primarily sensitive to antineutrinos, the recently developed Helium and Lead Observatory (HALO) is sensitive to neutrinos through charged current (CC) interaction mainly with ${ }^{208} \mathrm{~Pb}$ [21]. The hybrid method introduced in this work is based on the simultaneous implementation of two detectors, i.e., one for the neutrino and another one for the antineutrino signal from a supernova. Our model calculations show that complementary information from two respective detectors can provide constraints for the neutrino mass hierarchy.

From supernova simulations [22, 23] and the neutrino signal analysis of SN1987A [24, 25] we understand that (anti)neutrino fluxes detected from a distance have two well distinguished components, i.e., one on a short time-scale $(\lesssim 1 \mathrm{~s})$ due to emission from the accreting matter onto the proton-neutron star and a long-time scale component $(\approx 10 \mathrm{~s})$ due to Kelvin-Helmholtz cooling of the nascent proto-neutron star. Thus, being able to trace neutrino and antineutrino fluxes from ccSN provide a powerful tool to probe the dynamics of the stellar explosion but also various neutrino properties [26, 27]. In particular, the flavor content of the neutrino signal evolves due to both neutrino collective effects and matter effects which can lead to a highly interesting interplay of these and distinctive spectral features [30, 31]. In dense neutrino gas collective oscillations occur due to nonlinear flavor evolution phenomena associated to neutrino-neutrino interactions [32, 33]. The time-dependent fluxes from ccSN can be used to improve the understanding of the neutrino flavor transformations in regions of high neutrino densities [26 29, 34 38] and in transitions due to Mikheyev-Smirnov-Wolfenstein (MSW) effects occurring in the matter resonance layers of the stellar envelope 39 42]. More details on the neutrino flavor transformations in supernova are given in recent reviews [43, 44.

A microscopic description of neutrino-nucleus cross sections is crucial for the interpretation of the experimental data. It is also important to guide the design of new neutrino detectors with sufficient energy and time resolution (and also different thresholds), which are sensitive enough to register charged current events in the energy region $E_{\nu_{\mathrm{e}}\left(\bar{\nu}_{\mathrm{e}}\right)} \lesssim 30 \mathrm{MeV}$ from an intergalactic ccSN. In this particular energy region the largest difference of (anti)neutrino spectra between two possible neutrino mass hierarchies is expected, due to the impact of collective and MSW effects 26, 27, 38, 45].

Neutrino-nucleus interaction and cross sections in the energy region of supernova neutrinos have already been studied within a variety of microscopic approaches, including the shell model [46 [50], the random phase approximation (RPA) 51 54, continuum RPA (CRPA) 55 59], combined CRPA and shell model 51, 60 62, the Fermi gas model [63 65], quasiparticle RPA [66 70], projected QRPA 71] and relativistic quasiparticle RPA [2]. In Ref. [73] the cross sections for neutrino-induced particle emissions have been calculated for lead and iron neutrino detectors. Event rates from lead-based supernova-neutrino detectors have also been studied in Ref. [74], in order to explore the prospects for untangling the signatures of various oscillation scenarios.

In the present study, a microscopic theory framework based on the relativistic nuclear energy density functional and weak Hamiltonian is employed in the description of charged-current neutrino-nucleus reactions, including the properties of target nuclei, neutrino induced excitations, and weak interaction transition matrix elements [75, 76]. In order to account for the $\nu_{\mathrm{e}}\left(\bar{\nu}_{\mathrm{e}}\right)$ - induced events in the detector, the primary particle decay modes of the daughter nuclei are described, i.e., $\gamma$ decay and emission of one or two nucleons. Neutral current neutrino-nucleus reactions have not been taken into account in the present study. Their contributions in the neutron emission channel are small, less than $\approx 10 \%$ in heavy nuclei $\left[44\right.$. To determine positron related signals in water and mineral oil, $\bar{\nu}_{\mathrm{e}}$ cross sections of free protons were calculated in the framework introduced in Ref. [77, 78], based on chiral perturbation theory.

One of the main advantages of the hybrid method established in this work is the simultaneous detection of complementary parts of the neutrino spectra, i.e., $\nu_{\mathrm{e}}$ and $\bar{\nu}_{\mathrm{e}}$, in at least two different detectors, thus providing additional constraints as compared to traditional supernova analyses based mostly on antineutrino events in the detector. The time separation and characteristics of the fluxes from different supernova phases are used for the prediction of the detector events. Although the absolute number of (anti)neutrino events seen by the detector depends on the distance between supernova and Earth, the presented hybrid method is almost independent on this parameter and offers a promising approach for solving the problem of the neutrino mass hierarchy.

The paper is organized as follows. Section \includes the basic formalism and calculations of the (anti)neutrino fluxes incoming from an intergalactic ccSN, including the effects that depend on neutrino mass hierarchy. In Sec. III] we outline the nuclear models used for calculation of exclusive (anti)neutrino-nucleus cross sections and for the decay modes of daughter nuclei, i.e., one- and two-particle emissions together with $\gamma$ decay. In Sec. IV we provide further details on the actual hybrid method to determine the neutrino mass hierarchy. The analysis of the detector responses to supernova (anti)neutrinos and results of the hybrid method are presented in Sec. V] This includes a discussion of the role of uncertainties in supernova (anti)neutrino fluxes and their impact on reactions in different types of detectors. Section VI summarizes the results and includes an outlook for future studies. 


\section{SUPERNOVA NEUTRINOS}

Neutrinos and antineutrinos are produced at various stages of the ccSN evolution [79]. Model simulations result in several characteristic phases of neutrino emission, and provide the evolution of the (anti)neutrino luminosities and of the average energies 23, 79] : (i) luminosity rise during core collapse, (ii) shock breakout burst, (iii) accretion phase in which matter is still falling onto the proto-neutron star (from few tens to few hundreds ms after bounce), ending when neutrino heating reverses the infall, and (iv) Kelvin-Helmholtz cooling of the hot proto-neutron star with a duration of $10 \mathrm{~s}$ or more, accompanied by mass outflow in the neutrino-driven wind. The initial neutrino bursts (i),(ii) are unlikely to be used for improved neutrino-signal analysis due to the small number of detector events expected for average intergalactic supernova distances [22]. In the focus of the present study are neutrino induced responses from (anti)neutrinos associated to the accretion (iii) and cooling (iv) phases of ccSN.

During the accretion phase, the (anti)neutrino electron species with average energies $\left\langle E_{\nu_{e}}\right\rangle \approx 8-12 \mathrm{MeV}$ dominate [22, 23, 80], with roughly a factor of two larger luminosity than non-electron neutrinos in [22], while in [23] the difference is somewhat smaller. Within the cooling phase most of the energy released from the core leads to the creation of $\nu_{\mathrm{e}} \bar{\nu}_{\mathrm{e}}$ pairs, and neutrino luminosities may decrease by an order of magnitude. As shown in supernova simulation, in the cooling phase the luminosities of electron and non-electron species decrease with time evolution, and their differences are small [22, 23]. It is important to emphasize that the ordering of average energies remains the same through both phases, i.e., $\left\langle E_{\nu_{e}}\right\rangle \lesssim\left\langle E_{\bar{\nu}_{e}}\right\rangle \lesssim\left\langle E_{\nu_{x}}\right\rangle$. In this work, however, we will also explore deviations of luminosity ratios between different neutrino species to study the respective sensitivity of the detector response in Sec. V]

In the inner region of the star, up to several tens of km above the neutrinospheres, neutrino flavor conversion is first frozen by synchronization due to the strength of neutrino-neutrino interactions [37, 38. At larger distances from the neutrinospheres, the interaction strength decreases and bipolar oscillations occur [26, 27, 34, 37]. Neutrino-neutrino interactions provide a nonlinear term in the equations of motion [36, 38] and the result of such collective effects in the high-density neutrino region are spectral splits and swaps in the (anti)neutrino spectra. It is important to emphasize that recent studies of neutrino oscillations suggested that collective effects are strongly matter suppressed in the accretion phase [81, 82]. In the present analysis we include the three known neutrino species $\left(\nu_{\mathrm{e}}, \nu_{\mu}\right.$ and $\left.\nu_{\tau}\right)$, together with their antiparticles. We do not consider sterile types or a fourth generation.

The matter density profile of the exploding star changes drastically along the neutrino path. At a specific density, neutrino interactions with matter start dominating through forward elastic neutrino-electron scattering and the Mikheyev-Smirnov-Wolfenstein (MSW) effect induces efficient flavor transitions. There are two MSW resonance regions, the layer at higher densities (H-resonance layer) and lower densities (L-resonances layer) [41]. The neutrinospheres, however, are far below the resonance layers. In the neutrinosphere, the electron density is orders of magnitude larger than in the resonance layer. Thus, the flavor transition can simply be described by the LandauZener formula [41, 42]. In the iron core-collapse supernova the region dominated by collective effects and the two MSW resonant regions are spatially well separated (for neutrino energies above few $\mathrm{MeV}$ ) [41]. Therefore, probabilities for flavor transitions in different regions can be factorized [37]. For the accretion case, the transition probability is given by a $3 \times 3$ matrix, defined as

$$
P_{\mathrm{a}}=P_{\mathrm{MSW}}^{\mathrm{L}} P_{\mathrm{MSW}}^{\mathrm{H}},
$$

due to matter suppression of collective effects, and for the cooling phase we get

$$
P_{\mathrm{c}}=P_{\mathrm{MSW}}^{\mathrm{L}} P_{\mathrm{MSW}}^{\mathrm{H}} P_{\text {coll }},
$$

where $P_{\text {coll }}$ represents the flavor transition probability matrix at the end of the collective region, while the matrices $P_{\mathrm{MSW}}^{\mathrm{H}}$ and $P_{\mathrm{MSW}}^{\mathrm{L}}$ are related to conversions in the high and low matter density resonant regions, respectively, due to the MSW effect. In general, matrix elements are different for neutrinos and antineutrinos, and hierarchy dependent. They were calculated in Refs. [37, 41, 42]. The arrival of the supernova shock in the outer layers of the star can leave a mark on the $\nu_{\mathrm{e}}\left(\bar{\nu}_{\mathrm{e}}\right)$ spectra and even cause a non-adiabatic conversion and multiple MSW effects [37]. In addition to a multi-zenith angle instability [83], it has recently been shown that a multi-azimuth angle instability can occur in the case of normal mass hierarchy [84]. These effects were not taken into account in the supernova neutrino fluxes used here, i.e., a single-angle approximation was employed.

(Anti)neutrinos leave the surface of a star as mass eigenstates. On arrival at Earth, the incoming neutrino fluxes are incoherent due to spread of wave packets for typical supernova distances $(\approx 10 \mathrm{kpc})$, thus suppressing oscillations along the travelled distance $L$. In fact, they coincide with the neutrino fluxes on the surface of the star up to a geometrical factor $1 / L^{2}$. Few neutrinos, however, are detected as flavor states. The probability of detecting a particular flavor specie is given by

$$
P_{\mathrm{a}}^{\text {Earth }}=P_{\mathrm{PMNS}} P_{\mathrm{MSW}}^{\mathrm{L}} P_{\mathrm{MSW}}^{\mathrm{H}},
$$


for neutrinos emitted during the accretion phase, and

$$
P_{\mathrm{c}}^{\text {Earth }}=P_{\mathrm{PMNS}} P_{\mathrm{MSW}}^{\mathrm{L}} P_{\mathrm{MSW}}^{\mathrm{H}} P_{\text {coll }},
$$

for emission in the cooling phase. The matrix $P_{\mathrm{PMNS}}$ represents absolute squares of the Pontecorvo-Maki-NakagawaSakata matrix elements [1]. The above equations are applicable when the detector is directly turned to the neutrino source, minimizing Earth effects on the (anti)neutrino spectra. Therefore, the incoming fluxes are given by

$$
F_{\mathrm{a}(\mathrm{c})}^{\text {Earth }}=g(r) P_{\mathrm{a}(\mathrm{c})}^{\mathrm{Earth}} F_{\mathrm{a}(\mathrm{c})}^{\mathrm{init}},
$$

where $g(r)=R_{\nu_{\alpha}}^{2} / r^{2}$ is a geometrical factor depending on the supernova-Earth distance $r$. This is a general expression and can even be used with time-dependent (anti)neutrino fluxes. The initial energy distribution for each flavor is given by

$$
F_{\mathrm{a}(\mathrm{c}) ; \nu_{\alpha}}^{\mathrm{init}}\left(E_{\nu_{\alpha}}, R_{\nu_{\alpha}}\right)=\Phi_{\nu_{\alpha}}^{\mathrm{init}}\left(R_{\nu_{\alpha}}\right) \phi_{\nu_{\alpha}}\left(E_{\nu_{\alpha}}\right)
$$

where $\Phi_{\nu_{\alpha}}^{\text {init }}\left(R_{\nu_{\alpha}}\right)=L_{\nu_{\alpha}} /\left(4 \pi R_{\nu_{\alpha}}^{2}\left\langle E_{\nu_{\alpha}}^{0}\right\rangle\right)$ is the initial number flux of a particular species, $L_{\nu_{\alpha}}$ denotes the neutrino luminosity, and $R_{\nu_{\alpha}}$ corresponds to the radius of respective neutrinosphere. The initial neutrino spectra can be well described using a modified power-law distribution, also known as alpha-fit [85],

$$
\phi_{\nu_{\alpha}}\left(E_{\nu_{\alpha}}\right)=N\left(\frac{E_{\nu_{\alpha}}}{\left\langle E_{\nu_{\alpha}}^{0}\right\rangle}\right)^{\beta_{\nu}} \exp \left[-\left(\beta_{\nu}+1\right) \frac{E_{\nu}}{\left\langle E_{\nu_{\alpha}}^{0}\right\rangle}\right]
$$

with the normalization constant

$$
N=\frac{\left(\beta_{\nu}+1\right)^{\beta_{\nu}+1}}{\left\langle E_{\nu}^{0}\right\rangle \Gamma\left(\beta_{\nu}+1\right)},
$$

where $\Gamma$ denotes the Euler Gamma function, and $\beta_{\nu}$ is the pinching parameter. In general, $\beta_{\nu}$ is different for each neutrino species and varies in time. This parameter, however, is only weakly constrained by the SN1987A data. Only an average value for each phase of $\bar{\nu}_{\mathrm{e}}$ spectra can be derived from a fit to the data. Alternatively, theoretical results from supernova simulations (including the general time- and phase-dependence behavior) can be used instead. In this work, the alpha-fit distribution is employed, rather than the Fermi-Dirac distribution, due to its better description of the high energy tails of the spectra [86]. As shown in recent analysis of high-resolution neutrino spectra from a spherically symmetric supernova model [87], for the purpose of signal forecast in different detectors reasonably good accuracy can be achieved by considering the alpha-fit distribution. High energy tails of neutrino distributions are important because of the strong increase of neutrino-nucleus cross sections with neutrino energy and the possible dependence of neutrino-induced two-neutron emission rates in heavy nuclei on high-energy neutrino spectra. Such neutron emissions will be analyzed and discussed in Secs. III and V

Finally, we also define the relative luminosity as

$$
l_{\nu_{\alpha}}^{\mathrm{a}(\mathrm{c})}(\Delta t)=\frac{L_{\nu_{\alpha}}^{\mathrm{a}(\mathrm{c})}(\Delta t)}{L_{t o t}^{\mathrm{a}(\mathrm{c})}(\Delta t)},
$$

in order to avoid an explicit dependence on the total luminosity. In Sec. V we explore the sensitivity of the numbers of neutrino induced events in detectors and respective quantities introduced within the hybrid method, on the ratio of relative luminosities, $l_{\nu_{\mathrm{e}}}: l_{\bar{\nu}_{\mathrm{e}}}: l_{\nu_{\mathrm{x}}}$.

\section{III. (ANTI)NEUTRINO-NUCLEUS CROSS SECTIONS}

The charged-current (CC) neutrino- and antineutrino-nucleus reactions are considered in two steps, i.e., primary reactions

$$
\begin{aligned}
& \nu_{\alpha}+{ }_{Z} X_{N} \rightarrow{ }_{Z+1} X_{N-1}^{*}+l_{\alpha}^{-}, \\
& \bar{\nu}_{\alpha}+{ }_{Z} X_{N} \rightarrow{ }_{Z-1} X_{N+1}^{*}+l_{\alpha}^{+},
\end{aligned}
$$


and decay channels of the daughter nucleus in the case of Eq. (10a)

$$
{ }_{Z+1}^{A} X_{N-1}^{*} \rightarrow\left\{\begin{array}{l}
{ }_{Z+1}^{A} X_{N-1}+\gamma \\
A-1 \\
Z+1 \\
A-1 \\
A-2 \\
Z \\
A-4 \\
A-4 \\
Z-1 \\
A-2 \\
A-1 \\
Z+1
\end{array}\right.
$$

or in the case of Eq. (10b)

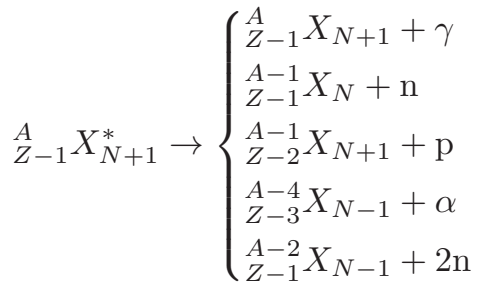

where $l_{\alpha}$ denotes the charged lepton (electron, muon, or its antiparticle). In this paper, however, we are mainly focused on electron species. The detector events following charged-current (CC) primary reactions with muon (anti)neutrinos are negligible for typical ccSN fluxes. The CC (anti)neutrino-nucleus cross section is given by [63, 88]

$$
\left(\frac{d \sigma_{\nu_{\alpha}\left(\bar{\nu}_{\alpha}\right)}}{d \Omega}\right)=\frac{1}{(2 \pi)^{2}} V^{2} p_{l} E_{l} \sum_{\substack{\text { lepton } \\ \text { spins }}} \frac{1}{2 J_{i}+1} \sum_{M_{i} M_{f}}\left|\left\langle f\left|\hat{H}_{W}\right| i\right\rangle\right|^{2}
$$

where $p_{l}$ and $E_{l}$ are the momentum and energy of the outgoing lepton, respectively, and $V$ is a normalization volume. The Hamiltonian $\hat{H}_{W}$ of the weak interaction is expressed in the standard current-current form, i.e., in terms of the nucleon $\mathcal{J}_{\lambda}(\boldsymbol{x})$ and lepton $j_{\lambda}(\boldsymbol{x})$ currents, as

$$
\hat{H}_{W}=-\frac{G}{\sqrt{2}} \int d \boldsymbol{x} \mathcal{J}_{\lambda}(\boldsymbol{x}) j^{\lambda}(\boldsymbol{x}),
$$

and the transition matrix elements are

$$
\left\langle f\left|\hat{H}_{W}\right| i\right\rangle=-\frac{G}{\sqrt{2}} l_{\lambda} \int d \boldsymbol{x} e^{-i \boldsymbol{q} \boldsymbol{x}}\left\langle f\left|\mathcal{J}^{\lambda}(\boldsymbol{x})\right| i\right\rangle .
$$

For the purpose of this work, the cross sections for the charged current $\nu_{e}\left(\bar{\nu}_{e}\right)$-nucleus reactions are calculated for the following target nuclei: ${ }^{12} \mathrm{C},{ }^{16} \mathrm{O},{ }^{56} \mathrm{Fe}$, and ${ }^{208} \mathrm{~Pb}$. The exclusive cross sections of the primary reactions are described in the framework based on the relativistic nuclear energy density functional (RNEDF) [72, 75, 76], by employing the density dependent effective interaction DD-ME2 89] in the particle-hole channel, while pairing correlations are described by the pairing part of the finite-range Gogny interaction with set D1S [90]. This framework has been successful in the description of Gamow-Teller transitions and other charge-exchange modes, thus it provides a reasonable method of choice to calculate relevant neutrino-induced transitions in nuclei [6, 91|. Transition matrix elements for neutrino-induced reactions are obtained using the general formalism from Refs. [63, 88]. This method allows to determine the ground state properties of target nuclei and transitions induced by neutrinos in a consistent way.

The exclusive cross sections are calculated as functions of excitation energy of the initial nuclei, including all contributions from the initial ground state of the even-even nucleus to the particular excited state of the daughter odd-odd nucleus, for all relevant multipolarities $J \leq 5$ and both parities. With the incoming (anti)neutrino fluxes in a terrestrial detector, the transition cross section to a particular state $i$ of the daughter nucleus is given by

$$
\sigma_{i}=\int_{E_{\nu_{\alpha}} \geq E_{\text {thresh }}} \sigma_{i}\left(E_{\nu_{\alpha}}\right) F_{\nu_{\alpha}}^{\text {Earth }}\left(E_{\nu_{\alpha}}\right) d E_{\nu_{\alpha}}
$$

and the flux averaged cross section is

$$
\left\langle\sigma_{i}\right\rangle=\frac{\sigma_{i}}{F_{\nu_{\alpha}}^{\text {tot }}}
$$


where the index $i$ denotes the excited state in the nuclear daughter with excitation energy $E_{i}^{\text {excit }}$, spin $J$, and parity $\pi$. The quantity $E_{\text {thresh }}$ is the energy threshold for a particular reaction (they usually vary from a few hundred keV to $\approx 10 \mathrm{MeV}$ ).

The primary reactions create daughter nuclei which de-excite through emission of photons or particles. Only these emissions can be registered in a terrestrial detector. Since we want to explore signatures of neutrino-induced reactions in such detectors, the emission channels of the daughter nuclei, as shown in Eqs. (11) and (12), have to be followed in a calculation. In this calculation we included all the channels shown in the above equations but here we particularly focus on neutron emission, as charged particle emission is negligible in comparison. The neutrino flux-integrated emission cross section $\sigma_{i \kappa}=\sigma_{i} P_{i \kappa}$ for daughter state $i$ and a particular channel $\kappa$ is simply the product of the flux-integrated cross section $\sigma_{i}$ and a branching ratio $P_{i \kappa}$, describing the relative contribution of channel $\kappa$ within all possible decay channels. This branching was calculated from the ratio of transmission coefficients $T_{i \kappa}$ obtained from solutions of the time-independent Schrödinger equation in the optical model,

$$
P_{i \kappa}=\frac{T_{i \kappa}}{\sum_{\kappa^{\prime}} T_{i \kappa^{\prime}}}, \kappa, \kappa^{\prime} \neq 2 \mathrm{n} .
$$

These transmission coefficients are related to reaction widths of resonances and are similar to those used in the Hauser-Feshbach model which describes compound nucleus reactions [92]. Therefore, we used the Hauser-Feshbach code SMARAGD [93, 94] to determine $P_{i \kappa}$. The procedure to calculate transmission coefficients is similar to the one described in 94, 95]. Emissions to final states include emissions to experimentally known discrete levels up to a final state excitation energy $E_{\text {fin }}$. Above $E_{\text {fin }}$ an integration over a nuclear level density for spins $J \leq 12$ and both parities is invoked. Thus, the calculated emission spectrum contains both discrete and continuous contributions, as is also typical for compound nucleus reactions. Here, the "compound" nucleus is formed by the primary CC weak reactions. The same default settings of the code were used as in 95] but with updated experimental levels (up to 40 known excited states from the 2010 version of NuDAT 96]) and level density (including parity dependence as described in 97]). Emission thresholds are given by the particle separation energies $S_{\kappa}$ in the emitting nucleus. These were computed from measured nuclear mass differences taken from [98]. It should be noted that the $\gamma$ cascade in the daughter was not followed in detail and therefore neutron emission after $\gamma$ deexcitation(s) is not included. We expect it to be negligible, anyway, because significant contributions to the total particle emissions only come from $\sigma_{i}$ with moderate excitation energy, $E_{i}^{\text {excit }} \lesssim 25 \mathrm{MeV}$, and separation energies are of the order of $10 \mathrm{MeV}$.

For emission of the second neutron, $T_{i \mathrm{n} 2}=T_{i \rightarrow j \mathrm{n}} \sum_{j_{i \rightarrow j}} P_{j \mathrm{n}}^{*}$ was calculated from a recursive procedure, starting from the transmission coefficient for one-neutron emission from state $i$ to final state $j, T_{i \mathrm{n}}$. For all final states $j$ possibly populated by emission of one neutron, the procedure of determining a branching ratio $P^{*}$ for single-neutron emission from the new daughter nucleus in state $j$ was repeated.

Using the above approach, it is not only possible to calculate flux-integrated cross sections for the individual particle emission channels but it is also possible to determine the emission spectrum $\mathcal{S}_{\kappa}\left(E_{\mathrm{em}}\right)$, i.e., the number of particles emitted with a given energy $E_{\mathrm{em}}$. This is useful if the detector cannot only provide an energy-integrated signal but also resolve the actual particle spectrum. To illustrate a first application of the hybrid method, however, in the following we restrict ourselves to predicting particle counts in the detector, i.e., energy integrated results. The detailed emission spectra are available from the authors on request and may provide further constraints in future analyses of neutrino signatures in detectors. Since the studies of lead detector efficiency are still in progress [99], in the present analysis perfect detector response is assumed, with the efficiency denoted by $\eta \rightarrow 1$.

The rate of particles registered in the detector is given by

$$
\frac{d N_{\kappa}}{d t}=N_{\mathrm{T}} D_{\kappa} \sum_{i} \sigma_{i} \widetilde{P}_{i \kappa}
$$

where in the case of emission of only one neutron (1n) from daughter nucleus we have $\widetilde{P}_{i 1 n}=P_{i 1 n}-P_{i 2 n}$, while for $\gamma$, one-proton (1p), two-neutron (2n) and emission of $\alpha$ particle $\widetilde{P}_{i \kappa}=P_{i \kappa}$. The total number of targets (nuclei or protons) in the detector is $N_{\mathrm{T}}=X m_{\mathrm{d}} N_{\mathrm{A}} / M_{r}$, where $m_{\mathrm{d}}$ denotes the detector mass, $X$ the number of particular targets (e.g., $X=2$ corresponds to the number of protons in water molecule, otherwise if no molecules are formed, $X=1), M_{r}$ the respective molar weight, and $N_{\mathrm{A}}$ the Avogadro constant (see Sec. IV for more details).

In addition to $\nu_{\mathrm{e}}\left(\bar{\nu}_{\mathrm{e}}\right)$-nucleus cross sections, in this work also inelastic $\bar{\nu}_{\mathrm{e}}$ scattering on free protons is taken into account. This is relevant for detectors using mineral oil (alkanes, which contain $\mathrm{CH}_{2}$ groups) or water $\left(\mathrm{H}_{2} \mathrm{O}\right)$. In the calculation, we employ heavy-baryon chiral perturbation theory, which also includes radiative corrections 77, 78]. The calculated cross sections for inelastic $\bar{\nu}_{\mathrm{e}}+\mathrm{p}$ scattering are shown in Fig. [1. In Sec $\mathrm{V}$, this cross section is employed in modeling the number of the detector events induced by antineutrinos in mineral oil and water. 


\section{DESCRIPTION OF THE HYBRID METHOD}

Predictions of the absolute values of the number of events for $\mathrm{NH}$ or IH may not provide conclusive information on the neutrino mass hierarchy due to the overlaps of the expected value ranges caused by the considerable uncertainties in the modeling of supernova (anti)neutrino fluxes [23]. In the following we introduce a hybrid method that could constrain the neutrino mass hierarchy. It is based on observing $\mathrm{CC}$ reactions with at least two detectors which cover complementary parts of supernova (anti)neutrino spectra, i.e., one detector is sensitive mainly to $\nu_{\mathrm{e}}$ while the other to $\bar{\nu}_{\mathrm{e}}$. At the core of the hybrid method is the definition of a set of hierarchy-dependent quantities, involving ratios of event numbers from several detectors and making use of different reactions as well as different phases of supernova evolution. In this way the dependence on the absolute values of some of the involved quantities is reduced, e.g., on the luminosities, on the supernova-Earth distance, and on the mass of the detector. In this work, we explore the feasibility to provide conclusive information on neutrino mass hierarchy by using the relative quantities of such a hybrid method based on two detectors, rather than depending on the absolute number of the events in a single detector.

In the analysis of time-dependent detector events, for each reaction channel the ratio of the numbers of emitted particles, both for the supernova accretion and cooling phase, is given by,

$$
R_{\kappa_{\mathrm{A}} \kappa_{\mathrm{B}}}^{\mathrm{a}(\mathrm{AB}}\left(\Delta t_{j}\right)=\frac{\sum_{\tau_{\mathrm{A}}} N_{\kappa_{\mathrm{A}}, \bar{\nu}_{\mathrm{e}}}^{\mathrm{a}(\mathrm{c})}\left(\Delta t_{j}\right)}{\sum_{\tau_{\mathrm{B}}} N_{\kappa_{\mathrm{B}}, \nu_{\mathrm{e}}}^{\mathrm{a}(\mathrm{c}) ; \tau_{\mathrm{B}}}\left(\Delta t_{j}\right)},
$$

and similarly its time-integrated variant. The indices A and B identify the detector with its particular decay or deexcitation channel $\kappa_{\mathrm{A}}, \kappa_{\mathrm{B}}$, of the daughter nucleus (see Sec. III). In addition, the labels $\tau_{A}$ and $\tau_{B}$ identify whether nuclei or free protons are involved in $\mathrm{CC}$ reactions in the detectors. For example, there are several different target nuclei in a water or mineral oil detector (free protons and isotopes of $\mathrm{C}$ or $\mathrm{O}$ ), which have different contributions to the number of $\mathrm{CC}$ events due to their different $\mathrm{CC}$ reaction cross sections. We assume that the signal of a particular emission mode can be well distinguished from signals of other modes. Furthermore, the time-bin $\Delta t_{j}$ should be wide enough to allow for good statistics of the events and it should be similar (comparable) for both detectors. If the $\nu-\nu$ interaction is sufficiently strong to cause collective effects in the region up to few hundred $\mathrm{km}$ above neutrinosphere, then flavor transitions will occur in complementary parts of the spectra, causing spectral splits and swaps. Furthermore, in the case of NH the MSW effect in both resonant regions will contribute to transitions between neutrinos, while the case of IH is characterized by transitions in the lower resonant region only. This is different, however, for antineutrinos which experience transitions in the low resonant region for $\mathrm{NH}$ and in both resonant regions for IH (for details, see [41]). The quantity $R_{\kappa \lambda}^{A B}$ is a relative measure of total supernova effects on initial (anti)neutrino spectra.

From Eqs. (19) and (20) we get

$$
R_{\kappa_{\mathrm{A}} \kappa_{\mathrm{B}}}^{\mathrm{a}(\mathrm{c}) \mathrm{AB}}\left(\Delta t_{j}\right)=\Lambda_{\mathrm{AB}} \frac{D_{\kappa_{\mathrm{A}}} \sum_{i, \tau_{\mathrm{A}}} X_{\mathrm{A}}^{\tau_{\mathrm{A}}} \sigma_{i\left(\bar{\nu}_{\mathrm{e}}\right)}^{\tau_{\mathrm{A}}}\left(\Delta t_{j}\right) \widetilde{P}_{i \kappa_{\mathrm{A}}}^{\tau_{\mathrm{A}}}}{D_{\kappa_{\mathrm{B}}} \sum_{i, \tau_{\mathrm{B}}} X_{\mathrm{B}}^{\tau_{\mathrm{B}}} \sigma_{i\left(\nu_{\mathrm{e}}\right)}^{\tau_{\mathrm{B}}}\left(\Delta t_{j}\right) \widetilde{P}_{i \kappa_{\mathrm{B}}}^{\tau_{\mathrm{B}}}},
$$

where

$$
\Lambda_{\mathrm{AB}}=\frac{\frac{m_{\mathrm{A}} N_{\mathrm{A}}}{M_{r}^{\mathrm{A}}}}{\frac{m_{\mathrm{B}} N_{\mathrm{A}}}{M_{r}^{\mathrm{B}}}}
$$

is constant for a given combination of neutrino (B) and antineutrino (A) detector. The coefficient $X_{\mathrm{A}(\mathrm{B})}^{\tau}$ stands for the number of targets (atoms or protons) in a molecule for mineral oil or water detectors. In the case of a detector using a metal, $X_{\mathrm{A}(\mathrm{B})}^{\tau}=1$. The relative molecular $(\mathrm{A})$ or atomic (B) molar weight is denoted by $M_{r}^{\mathrm{A}(\mathrm{B})}$. Note that even in a single detector several targets can give contributions to the total $\gamma, 1 \mathrm{n}, 1 \mathrm{p}$ and $2 \mathrm{n}$ emissions. In Eq. (21) one can see that $R_{\kappa_{\mathrm{A}} \kappa_{\mathrm{B}}}^{\mathrm{AB}}$ contains three types of information, the coefficient $\Lambda_{\mathrm{AB}}$ depends only on the combination of detectors used, the coefficients $D_{\kappa}$ and $\widetilde{P}_{i \kappa}^{\tau}$ are connected to the particle emission and its probability of observation, and third quantity $\sigma_{i}^{\tau}$, which is the transition probability at a given neutrino flux per target nucleus. The latter term is rather complex as it depends on the structure of the nuclear ground and excited states, and on the (anti)neutrino spectra. Thus, it also contains imprints of collective and MSW effects, which are generally different for NH and IH.

In order to eliminate the dependence on the particular detector combination, thus using only information on particular targets involved in CC reactions for $\eta \rightarrow 1$ (perfect detector response), we define the reduced quantity,

$$
r_{\kappa_{\mathrm{A}} \kappa_{\mathrm{B}}}^{\mathrm{AB}}=\lim _{\eta \rightarrow 1} R_{\kappa_{\mathrm{A}} \kappa_{\mathrm{B}}}^{\mathrm{AB}} / \Lambda_{\mathrm{AB}}
$$


which is an effective ratio of transformation probabilities (including decay modes) per single atom (B) or molecule (A) caused by complementary parts of the incoming supernova neutrino fluxes. The quantity $r_{\kappa_{\mathrm{A}} \kappa_{\mathrm{B}}}^{\mathrm{AB}}$ still contains information about targets included in the processes, and on the empirical structure for molecules when dealing with water and mineral oil. In other words, the reduced quantity $r_{\kappa_{\mathrm{A}} \kappa_{\mathrm{B}}}^{\mathrm{AB}}$ is good measure for hierarchy-dependent effects, i.e., a relative measure of the effects that shape (anti)neutrino spectra, independent of the absolute values of detector mass, effective number of targets, and explicit supernova-Earth distance.

\section{RESULTS}

The results of the present analysis are twofold: the first category is related to the detector response to the fluxes of supernova (anti)neutrinos, i.e., the induced particle emissions from target nuclei; the second is related to applications of the hybrid method to constrain the neutrino mass hierarchy.

\section{A. Supernova (anti)neutrino fluxes}

In order to investigate the feasibility of the hybrid method despite of the current uncertainties in predicting neutrino fluxes [23], the effects of various sets of initial supernova flux parameters are explored. We used several combinations of initial luminosities in the accretion $\left(\sim 10^{52} \mathrm{erg} / \mathrm{s}\right)$ and cooling phase $\left(\sim 10^{51} \mathrm{erg} / \mathrm{s}\right)$ fluxes, with a fixed total emitted energy for each phase (see Tab. I), i.e., $L_{\mathrm{tot}}^{\mathrm{acc}} \approx 0.4 \times 10^{53} \mathrm{erg}$ for a duration of $0.4 \mathrm{~s}$, and $L_{\mathrm{tot}}^{\text {cool }} \approx 2 \times 10^{53} \mathrm{erg}$ for a duration of $10 \mathrm{~s}$. We also varied the initial average energies of neutrinos from 8 to $12 \mathrm{MeV}$ for $\nu_{\mathrm{e}}$, from 11 to $15 \mathrm{MeV}$ for $\bar{\nu}_{\mathrm{e}}$, and from 11 to $19 \mathrm{MeV}$ for non-electron species. Thus, the range of values from different simulations of supernova neutrino fluxes is covered [22, 23, 38], and the sensitivity of the hybrid method on the differences in neutrino fluxes can be explored. In the present analysis, the initial averaged energies of neutrino species were kept in the canonical order, as shown in Sec. II with two additional conditions: $\left\langle E_{\bar{\nu}_{\mathrm{e}}}^{0}\right\rangle-\left\langle E_{\nu_{\mathrm{e}}}^{0}\right\rangle \lesssim 3 \mathrm{MeV}$ and $\left\langle E_{\nu_{n}}^{0}\right\rangle-\left\langle E_{\bar{\nu}}^{0}\right\rangle \lesssim 4$ $\mathrm{MeV}$. The pinching parameter $\beta_{\nu}$ in Eq.(17) was set to 4.0 for the accretion and 3.0 for the cooling phase fluxes [38, 85]. In calculating transition probabilities beyond the collective region, we have used fixed values of splitting energies from a supernova neutrino flux analysis [80]. The incoming (anti)neutrino fluxes were calculated including collective and MSW effects in the core-collapsing star (see Sec. II). Model calculations include best-fit values of neutrino oscillation parameters. They can be found, e.g., in [45], although somewhat different values are given in [100, 101]. As a test case for ccSN in our galaxy, an imaginary star 25000 l.y. away from Earth was assumed.

As an example, Fig. 2 shows the set of incoming $\nu_{e}$ and $\bar{\nu}_{e}$ fluxes of the accretion and cooling supernova phases for the normal $(\mathrm{NH})$ and inverted mass hierarchy $(\mathrm{IH})$. Three combinations of the luminosity ratios of (anti)neutrino species for type II supernova spectra in accretion(A1-A3) and cooling (C1-C3) phase are considered, as given in Tab. I The initial average energies of the supernova fluxes shown in Fig. 2 are $\left\langle E_{\nu_{\mathrm{e}}}^{0}\right\rangle=10 \mathrm{MeV},\left\langle E_{\bar{\nu}_{\mathrm{e}}}^{0}\right\rangle=13 \mathrm{MeV}$, and $\left\langle E_{\nu_{x}}^{0}\right\rangle$ $=15 \mathrm{MeV}$.

\section{B. Nuclear responses to supernova (anti)neutrinos}

Figures 3 and 4 show examples of the CC primary cross sections of ${ }^{56} \mathrm{Fe}$ averaged over supernova cooling phase fluxes of $\nu_{\mathrm{e}}$ and $\bar{\nu}_{\mathrm{e}}$ (for the case C3 in Tab. II), respectively, and displayed as a function of excitation energy in the initial nucleus. Contributions to the cross sections are shown separately for several multipoles, from $J^{\pi}=0^{ \pm}$to $J^{\pi}=3^{ \pm}$. The energy threshold for CC reactions in ${ }^{56} \mathrm{Fe}$ is relatively low for $\nu_{\mathrm{e}}(4.57 \mathrm{MeV})$ and $\bar{\nu}_{\mathrm{e}}(4.72 \mathrm{MeV})$. The $\nu_{\mathrm{e}}{ }^{56} \mathrm{Fe}$ cross section is mainly determined by $J^{\pi}=0^{+}, 1^{+}$transitions, while the contribution of $J^{\pi}=1^{-}, 2^{-}$states is an order of magnitude smaller. Other multipolarities contribute only marginally. As shown in the upper panel of Fig. 4, the $\bar{\nu}_{\mathrm{e}^{-}}{ }^{56} \mathrm{Fe}$ reaction cross section, on the other hand, is dominated by $J^{\pi}=1^{+}$transitions, with a major contribution from the excited state at $4.5 \mathrm{MeV}$. The lower panels of Figs. 3 and 4 show the ratio of flux averaged cross sections between two hierarchies for the specific incoming cooling phase $\nu_{\mathrm{e}}$ or $\bar{\nu}_{\mathrm{e}}$ flux C3. They indicate that exclusive transitions to particular states should be reduced in the case of IH by factors $\approx 1.1-2.15$ for $\nu_{\mathrm{e}}$ and $\approx 1.15-1.32$ for $\bar{\nu}_{\mathrm{e}}$. A similar effect is expected for the other flux cases.

The total $\nu_{\mathrm{e}}$-nucleus CC reaction cross section is increasing rapidly with increasing number of neutrons in the nucleus [75]. The relative difference in the depths of the proton and the neutron part of the mean field potential increases in nuclei with neutron excess, however, Pauli blocking strongly suppresses transitions from proton to neutron quasiparticle states in $\bar{\nu}_{\mathrm{e}}$-nucleus reactions. This effect is evident in the $\bar{\nu}_{\mathrm{e}^{56}}{ }^{5 \mathrm{Fe}}$ reaction, where the cross sections are an order of magnitude smaller than in the case of neutrino induced reaction (see Figs. 3 and 4 ), although ${ }^{56} \mathrm{Fe}$ has a relatively small neutron excess $(N-Z=4)$. The upper panel of Fig. 5 shows the exclusive cross sections for the 
$\nu_{\mathrm{e}}{ }^{208} \mathrm{~Pb}$ reaction, illustrating an example of a very neutron-rich target. The overall cross sections are considerably larger than for ${ }^{56} \mathrm{Fe}$, thus indicating that a lead-based detector is a reasonable choice to efficiently detect supernova neutrinos. On the other hand, due to the strong blocking effect in the single-particle spectra, $\bar{\nu}_{\mathrm{e}^{-}}{ }^{208} \mathrm{~Pb}$ cross sections are considerably reduced, i.e., the lead detector allows measurements of supernova-neutrino induced events only.

\section{Detector responses to supernova (anti)neutrinos}

By employing the framework outlined in Sec. III] we study neutrino induced events in detectors based on various target material. Since perfect efficiency is assumed, we note that in the realistic case the observed number of the events may considerably reduce, e.g., the efficiency to detect a neutron generated in a lead-based detector can be as low as a few tens of percent [99, 102. Furthermore, it is assumed that the detector is turned directly to the incoming neutrino flux, thus eliminating Earth effects on neutrino spectra. Four cases of target material were considered, mineral oil $\left(\mathrm{CH}_{2}\right)$, water $\left(\mathrm{H}_{2} \mathrm{O}\right),{ }^{56} \mathrm{Fe}$, and ${ }^{208} \mathrm{~Pb}$. The mass of the detector was taken to be $1 \mathrm{kt}$ for all cases of target material.

The numbers of (anti)neutrino-induced events due to $1 \mathrm{n}, 2 \mathrm{n}$, and $e^{+}$emissions for several different accretion supernova fluxes are shown in Tab. II both for normal and inverted neutrino mass hierarchies. Three combinations of the luminosity ratios (A1,A2,A3) are considered, as given in Tab. II In addition, three sets of the initial average energies of the supernova fluxes are used, (i) $\left\langle E_{\nu_{\mathrm{e}}}^{0}\right\rangle=8 \mathrm{MeV},\left\langle E_{\bar{\nu}_{\mathrm{e}}}^{0}\right\rangle=11 \mathrm{MeV},\left\langle E_{\nu_{x}}^{0}\right\rangle=13 \mathrm{MeV}$; (ii) $\left\langle E_{\nu_{\mathrm{e}}}^{0}\right\rangle=10$ $\mathrm{MeV},\left\langle E_{\bar{\nu}_{\mathrm{e}}}^{0}\right\rangle=13 \mathrm{MeV},\left\langle E_{\nu_{x}}^{0}\right\rangle=15 \mathrm{MeV}$; and (iii) $\left\langle E_{\nu_{\mathrm{e}}}^{0}\right\rangle=12 \mathrm{MeV},\left\langle E_{\bar{\nu}_{\mathrm{e}}}^{0}\right\rangle=15 \mathrm{MeV},\left\langle E_{\nu_{x}}^{0}\right\rangle=19 \mathrm{MeV}$. The number of $\bar{\nu}_{\mathrm{e}}$-related events, i.e., the number of emitted positrons, is expected to depend not only on the initial average energies of the $\nu$ spectra, but also on the initial luminosity ratios between neutrino species. Due to large overlap of the respective positron events, however, it is difficult to put any constraint on the neutrino mass hierarchy from these events alone.

The number of single-neutron emissions in ${ }^{56} \mathrm{Fe}\left({ }^{208} \mathrm{~Pb}\right)$ is $\approx 10$ (few) times smaller than positron events related with free protons in water and mineral oil, while two-neutron events in ${ }^{208} \mathrm{~Pb}$ are negligible for cases (i) and (ii). The number of single-neutron events in ${ }^{56} \mathrm{Fe}$ and ${ }^{208} \mathrm{~Pb}$ significantly increases with the initial average energies of the $\nu$ spectra. The number of emitted neutrons, however, remains ambiguous for the shown cases, due to the strong matter suppression of collective effects, which was also observed in [37. Therefore, the analysis of the detector response to the (anti)neutrinos from the accretion phase does not allow to distinguish between the two neutrino mass hierarchies.

Table III shows the number of detector events in the same target materials as discussed above, but for the (anti)neutrino fluxes from the cooling phase, for the luminosity ratios in Tab. I denoted as C1,C2,C3. Again, three sets of the initial average energies of the supernova fluxes are considered. The number of positron events in water or mineral oil induced by $\bar{\nu}_{\mathrm{e}}$ is at least a few times larger than the number of primary $\nu_{\mathrm{e}}$ reactions with ${ }^{56} \mathrm{Fe}$ and ${ }^{208} \mathrm{~Pb}$ for all investigated cooling phase neutrino fluxes (with different values of initial average energies as shown in the table). The number of single-neutron emissions for ${ }^{56} \mathrm{Fe}$ and for one- and two-neutron emissions for ${ }^{208} \mathrm{~Pb}$ target is larger in the case of NH (see Figs. 3 and 5). Contrary to the accretion phase, the neutrino fluxes from the cooling phase result in an increased number of the events for $\mathrm{NH}$ and thus in a reasonable separation between the numbers of the particle emissions from nuclei for the two hierarchies.

The most pronounced response to $\mathrm{CC}$ interactions with $\nu_{\mathrm{e}}$ is obtained for ${ }^{208} \mathrm{~Pb}$. Due to large number of protons $(Z=82)$, Coulomb effects enhance the phase space for emitted electrons [103]. It also has large neutron excess $(N-Z=44)$ and relatively low threshold $(2.88 \mathrm{MeV})$ for CC reactions. For the supernova cooling phase, we obtained a flux averaged cross section for neutron emission from ${ }^{208} \mathrm{Bi}$ of $\approx 10^{-40} \mathrm{~cm}^{2}$. In addition, a significant contribution of two-neutron events was obtained due to relatively high two-neutron cross sections $\left(\approx 10^{-41}-10^{-40} \mathrm{~cm}^{2}\right)$ at energies above the two-neutron separation energy of $23 \mathrm{MeV}$ relative to the mother nucleus (see upper panel of Fig. 5). The same applies for all sets (i)-(iii) of neutrino fluxes. The sensitivity of the number of 2 n-emissions on the type of mass hierarchy is evident in the cooling phase, i.e., the ratio $N_{2 \mathrm{n}}^{\mathrm{NH}} / N_{2 \mathrm{n}}^{\mathrm{IH}} \approx 2$ (see lower panel of Fig. 5 and Tab. III) ).

The cross sections for $\bar{\nu}_{\mathrm{e}}-{ }^{208} \mathrm{~Pb}$ reactions are strongly suppressed (by three orders of magnitude) due to Pauli blocking of the neutron single-particle states. Thus, lead presents an excellent choice for a supernova neutrino detector to be used in the hybrid method. In order to obtain good statistics of the events, though, a sufficient amount of target material has to be available, e.g., $\approx 1 \mathrm{kt}$ when assuming typical intragalactic supernova-Earth distances. The few reactions with $\bar{\nu}_{\mathrm{e}}$ and $\nu_{\mu}$ in this material can be neglected in comparison to $\nu_{\mathrm{e}}$ related events. Moreover, natural lead has a comparatively small thermal neutron capture cross section of $\approx 0.15$ barn, which allows lead to moderate fast neutrons almost without absorption prior to arrival at a neutron counter [104]. Although lead isotopes have large neutron excess, some of the neutrons might still be captured by ${ }^{206} \mathrm{~Pb}$ and ${ }^{207} \mathrm{~Pb}$, though [105].

In comparison, ${ }^{56} \mathrm{Fe}$ target is characterized by somewhat weaker response to $\mathrm{CC}$ reactions with neutrinos (see Tab. II and Tab. III for the accretion and cooling phase neutrinos, respectively). The predicted total cross section of neutrino-induced reactions in ${ }^{56} \mathrm{Fe}$ is $\approx 10$ times smaller than for ${ }^{208} \mathrm{~Pb}$. Almost half of the predicted events come from single-neutron emission from ${ }^{56} \mathrm{Co}$, while two-neutron emissions are severely reduced. Due to small neutron 
excess $(N-Z=4)$, the detector based on ${ }^{56} \mathrm{Fe}$ is also sensitive to antineutrino CC reactions with cross sections of $\approx 10^{-42} \mathrm{~cm}^{2}$, an order of magnitude smaller than for $\nu_{\mathrm{e}}-{ }^{56} \mathrm{Fe}$ reactions, but the daughter nuclei mostly deexcite by emission of $\gamma$ rays. Furthermore, the cross sections for absorption of thermal neutrons in natural iron is an order of magnitude larger than in lead $(\approx 2.5$ barn $[104])$, and thus iron appears to be an inappropriate target material for a $\nu_{\mathrm{e}}$ and $\bar{\nu}_{\mathrm{e}}$ detector.

For mineral oil (water) with a density of $0.85 \mathrm{~g} / \mathrm{cm}^{3}\left(1.0 \mathrm{~g} / \mathrm{cm}^{3}\right.$ at $\left.4{ }^{\circ} \mathrm{C}\right)$, in which the target nuclei are ${ }^{12} \mathrm{C}\left({ }^{16} \mathrm{O}\right)$, the predicted cross sections are of the order of $\approx 10^{-42} \mathrm{~cm}^{2}$. Due to $N=Z=6(N=Z=8)$, the difference in total number of primary events between neutrino and antineutrino CC reactions is smaller than for $N>Z$ nuclei. Due to relatively high energy thresholds $(\gtrsim 11 \mathrm{MeV})$ for both types of reactions in ${ }^{12} \mathrm{C}\left({ }^{16} \mathrm{O}\right)$, the expected responses are rather low for $\nu_{e}\left(\bar{\nu}_{e}\right)$ energies of $\lesssim 20 \mathrm{MeV}$. Actually, in these detectors $\bar{\nu}_{\mathrm{e}}$ induce reactions mainly with free protons (see Tab. II and Tab. III), with a low energy threshold $(1.8 \mathrm{MeV})$ and cross sections $\sigma \approx 10^{-43}\left(E_{\nu} / \mathrm{MeV}\right)^{2}$. The dominance of $\bar{\nu}_{\mathrm{e}}-\mathrm{p}$ reactions in mineral oil and water ensures an efficient coverage of the $\bar{\nu}_{\mathrm{e}}$ spectra. Therefore, detectors based either on mineral oil or water can cover the antineutrino response in the hybrid method. In these cases the influence of CC reactions of $\nu_{\mathrm{e}}$ and $\bar{\nu}_{\mathrm{e}}$ with nuclei amounts only $\approx 1 \%$ in the response and thus CC events with nuclei can be neglected. Similar results were obtained in Ref. [106], i.e., only $\approx 1.5 \%(5.6 \%)$ of the reactions are expected to be related to ${ }^{16} \mathrm{O}\left({ }^{12} \mathrm{C}\right)$ in $\mathrm{CC}$ and $\mathrm{NC}$ reactions in the KII detector (Large Volume Detector) in the energy range of interest. An additional $\gamma$ signal related to neutron capture in the detector (either on the original target nuclei or after adding an impurity such as Gd to increase the capture rates) can be used to confirm or eliminate charge exchange $\bar{\nu}_{\mathrm{e}}-\mathrm{p}$ events [107].

\section{Application of the hybrid method}

In the following we present calculations of the ratios of $\bar{\nu}_{e}$ and $\nu_{e}$ detector events for a variety of possible incoming supernova (anti)neutrino accretion and cooling phase fluxes (see Tab. \), both for normal (NH) and inverted (IH) mass hierarchy. By using $r_{\kappa_{\mathrm{A}} \kappa_{\mathrm{B}}}^{\mathrm{AB}}$ as defined in Eq. (23), we obtained mass and distance invariant values for the most sensitive decay channels in the detectors. Statistical uncertainties in $r_{\kappa_{\mathrm{A}} \kappa_{\mathrm{B}}}^{\mathrm{AB}}$, however, still depend on such quantities as distance, etc. Due to the reasons given above, we eliminated ${ }^{56} \mathrm{Fe}$ from the further analysis and used only ${ }^{208} \mathrm{~Pb}$ and mineral oil (water) for the neutrino and antineutrino sectors, respectively. Including only dominant channels in the analysis, the quantity $r_{\kappa_{\mathrm{A}} \kappa_{\mathrm{B}}}^{\mathrm{AB}}$ should be the same in the $\mathrm{Pb} / \mathrm{H}_{2} \mathrm{O}$ and $\mathrm{Pb} / \mathrm{CH}_{2}$ detector combinations, due to the similarity of structural (empirical) formula of water and mineral oil with 2 free protons each.

In Fig. 6 the following ratios $r_{\kappa_{\mathrm{A}} \kappa_{\mathrm{B}}}^{\mathrm{AB}}$ of the detector events are shown for the incoming (anti)neutrino fluxes of the accretion phase for $\mathrm{NH}$ and $\mathrm{IH}$, including statistical uncertainties: (a) $r_{\mathrm{e}^{+}+\mathrm{n}}^{\text {free }, \mathrm{Pb}}$ (b) $r_{\mathrm{e}^{+} 2 \mathrm{n}}^{\text {free }, \mathrm{Pb}}$, and (c) $r_{\mathrm{e}^{+} \text {tot } \mathrm{n}}^{\text {free }, \mathrm{Pb}}$. The quantities (a), (b), and (c) correspond to the ratios between the numbers of positron events in the antineutrino detector (with dominant $\bar{\nu}_{\mathrm{e}}$-p events) and neutrino-induced one-, two-, and total neutron emissions in the Pb detector, respectively. We employed three combinations of the luminosities for the accretion phase (A1-A3) for three different configurations of initial average energies of neutrino spectra, as specified in Tab. I.

As seen in Fig. 6. the ratios of $\bar{\nu}_{\mathrm{e}}$ and $\nu_{\mathrm{e}}$ induced events result in reasonable separation between $\mathrm{NH}$ and IH for the A2 case, which has a larger asymmetry in relative luminosities between electron and non-electron species, independent of initial average neutrino energies. The other cases of neutrino fluxes are characterized by partial overlaps between $\mathrm{NH}$ and IH event ratios for all energy configurations. By considering other ratios shown in Fig. 6] we note that neutrinos from the accretion phase systematically do not provide conclusive information to determine the mass hierarchy, with the exception of flux $A 2$.

In Fig. 7 we show the same decay channel analysis as in Fig. 6, but for the neutrino fluxes of the supernova cooling phase. Three types of the cooling phase (anti)neutrino fluxes were considered (C1-C3), as given in Tab. I and the same energy configurations as for the accretion fluxes given above. As seen in the figure, the (anti)neutrino induced events for the cooling phase are characterized by complete separation of the ranges of values calculated for $\mathrm{NH}$ and

$\mathrm{IH}$ : (a) $r_{\mathrm{e}^{+} 1 \mathrm{n}}^{\text {free }} \mathrm{Pb},(\mathrm{b}) r_{\mathrm{e}^{+} 2 \mathrm{n}}^{\text {free }, \mathrm{Pb}}$, and (c) $r_{\mathrm{e}^{+} \text {tot } \mathrm{n}}^{\text {free }}$ for all cases of supernova neutrino fluxes. Since the detectors with target material based on heavy nuclei are usually used only as counters, the number of emitted neutrons can provide additional information for the $\nu_{\mathrm{e}}$ part of spectra. Nevertheless, the strong dependence of $\mathrm{e}^{+}$, one- and two-neutron emissions on the average initial energies of $\nu$ can be used to constrain their initial energy configurations (see Tab. III).

The results of the present analysis also display sensitivity to the degree of asymmetry of the initial relative luminosities between $\nu$ species. In the case of $\mathrm{C} 2$ (larger $l_{\nu}$ asymmetry), the $r$-quantities are systematically shifted toward smaller values (see Fig. 7). In the case of two-neutron emissions, a complete distinction of the $r_{\mathrm{e}^{+}, 2 \mathrm{n}}^{\text {free } \mathrm{Pb}}$ quantities between two hierarchies is obtained, similar as in the case of one-neutron emissions. Therefore, in a detector with sensitivity to one- and two-neutron emissions, such as HALO, both types of the events can be used to constrain the neutrino mass hierarchy. We note that while this may be feasible for the (ii) and (iii) cases of supernova neutrino 
energy configurations, in the (i) case the number of two-neutron events is rather small (see Tab. III). Thus, it would be difficult to provide reliable limits. Nevertheless, the separation of the $r$-quantities as shown in Fig. 7 especially in the case of a reasonable number of single-neutron and total neutron emission events (Tab. III), clearly demonstrates the promise in using the hybrid method to constrain the neutrino mass hierarchy. Additional, currently not available, information on the sensitivity and efficiency of the HALO detector would allow a further, more detailed feasibility study for the proposed method.

\section{CONCLUSION}

We have presented a hybrid method to determine the neutrino mass hierarchy by simultaneous measurements of supernova $\nu_{\mathrm{e}}$ and $\bar{\nu}_{\mathrm{e}}$ events in detectors based on different types of target material. Using supernova $\nu_{\mathrm{e}}\left(\bar{\nu}_{\mathrm{e}}\right)$ fluxes that include collective and MSW effects for the accretion and cooling phases, responses in mineral oil, water, ${ }^{56} \mathrm{Fe}$ and

${ }^{208} \mathrm{~Pb}$ have been analyzed both for normal and inverted neutrino mass hierarchies. The analysis of charge-exchange excitation spectra for ${ }^{56} \mathrm{Fe}$ and ${ }^{208} \mathrm{~Pb}$ targets demonstrates the sensitivity of the nuclear response to the neutrino mass hierarchy, that is different for various multipole transitions. The hybrid method, that combines antineutrino-induced events in water or mineral oil with neutrino-induced emissions in heavier target nuclei (such as ${ }^{208} \mathrm{~Pb}$ ), could provide a useful tool to constrain the neutrino mass hierarchy. The number of emitted neutrons for NH in general is larger than for $\mathrm{IH}$, both for ${ }^{208} \mathrm{~Pb}$ and ${ }^{56} \mathrm{Fe}$ target nuclei. Since it is rather difficult to compare the absolute values of calculated particle emissions with the detector events, a set of hierarchy dependent relative quantities is introduced, which are independent of the supernova-Earth distance, of the effective number of targets in $\nu_{\mathrm{e}}$ and $\bar{\nu}_{\mathrm{e}}$ detectors, of their total masses, and of the ratios of these quantities. They contain the information on nuclear structure, excited states, relevant charge-exchange transitions and particle emission modes induced in nuclei by supernova (anti)neutrinos. The incoming (anti)neutrino fluxes employed in this study include the imprints of collective and MSW effects during the ccSN event and these are generally different between NH and IH. Model calculations show that the ratio of the numbers of $\nu_{\mathrm{e}}$ and $\bar{\nu}_{\mathrm{e}}$ induced detector events represents a quantity that allows to distinguish between the two neutrino mass hierarchies. Simultaneous comparison of several ratios of the numbers of detector events associated to various emitted particles for different supernova phases would reduce the uncertainties that may arise by considering only a single quantity.

As confirmed in calculations, heavy nuclei are almost completely inert to $\mathrm{CC}$ reactions with antineutrinos due to the strong Pauli blocking of neutron single-particle states. The pure neutrino signal and large CC cross section for reactions with neutrinos establish ${ }^{208} \mathrm{~Pb}$ as probably the most important nuclear target for neutrino detection and reconstruction of the neutrino part of spectra. On the other side, detectors based on mineral oil or water as target-material represent the most reasonable choice for $\bar{\nu}_{e}$ detection. Taking into account current neutrino detector developments (in particular, HALO $\left({ }^{208} \mathrm{~Pb}\right)$ [21], Super-Kamiokande (water) [108], etc.), the hybrid method presented in this work will provide a useful tool to constrain the neutrino mass hierarchy when the next galactic ccSN appears.

\section{Acknowledgments}

We thank Kate Scholberg, Meng-Ru Wu, and Maik Frensel for the information and comments of relevance for the paper. This work has been supported in part by FP7-PEOPLE-2011-COFUND program NEWFELPRO, the Swiss National Science Foundation, the Croatian Science Foundation under the project Structure and Dynamics of Exotic Femtosystems (IP-2014-09-9159), and by Deutscher Akademischer Austausch Dienst (DAAD). T.R. acknowledges support from the European Research Council (grant GA 321263-FISH) and the British STFC grant ST/M000958/1.

[1] Z. Maki, M. Nakagawa, and S. Sakata, Prog. Theor. Phys. 28, 870 (1962).

[2] B. Pontecorvo, Zh. Eksp. Teor. Fiz. 53, 1717 (1967) [Sov. Phys. JETP 26, 984 (1968)].

[3] M. C. Gonzalez-Garcia and M. Maltoni, Phys. Rep. 460, 1 (2008).

[4] A. Strumia and F. Vissani, arXiv:hep-ph/0606054 (2010).

[5] S.T. Petcov, M. Piai, Phys. Lett. B 533, 94 (2002).

[6] Yu-Feng Li, Jun Cao, Yifang Wang, and Liang Zhan, Phys. Rev. D 88, 013008 (2013).

[7] F. Capozzi, E. Lisi, and A. Marrone, Phys. Rev. D 89, 013001 (2014).

[8] M. Ishitsuka, T. Kajita, H. Minakata, H. Nunokawa, Phys. Rev. D 72033003 (2005).

[9] C. Lunardini and A. Yu Smirnov, J. Cosm. Astropart. Phys. 06, 009 (2003).

[10] B. Dasgupta, A. Dighe, A. Mirizzi, Phys. Rev. Lett. 101171801 (2008). 
[11] H. Duan, G. M. Fuller, J. Carlson, Y. Z. Qian, Phys. Rev. Lett. 99, 241802 (2007).

[12] P. D. Serpico, S. Chakraborty, T. Fischer et al., Phys. Rev. D 85, 085031 (2012).

[13] G. J. Mathews, T. Kajino, W. Aoki, W. Fujiya, and J. B. Pitts, Phys. Rev. D 85, 105023 (2012).

[14] W. Winter, Phys. Rev. D 88, 013013 (2013).

[15] Georg G. Raffelt, Nucl. Phys. B (Proc. Suppl.) 221, 218 (2011).

[16] K. Hirata et al. (KAMIOKANDE-II Collaboration), Phys. Rev. Lett. 58, 1490 (1987).

[17] R. M. Bionta et al., Phys. Rev. Lett. 58, 1494 (1987).

[18] E. N. Alekseev, L. N. Alekseeva, I. V. Krivosheina and V. I. Volchenko, Phys. Lett. B 205, 209 (1988).

[19] K. Scholberg, Proc. Neutrino 2006, arXiv:astro-ph/0701081

[20] K. Scholberg, J. Phys.: Conf. Series 203, 012079 (2010).

[21] C. Duba, F. Duncan, J. Farine, et al., J. Phys. Conf. Ser. 136, 042077 (2008).

[22] T. Fischer, S. C. Whitehouse, A. Mezzacappa, F.-K. Thielemann, and M. Liebendörfer, Astr. Astrophys., 517, A80 (2010).

[23] L. Hüdepohl, B. Muller, H. T. Janka, A. Marek, G. G. Raffelt, Phys. Rev. Lett. 104, 251101 (2010).

[24] T. J. Loredo, D. Q. Lamb, Phys. Rev. D 65, 063002, (2002).

[25] F. Vissani, G. Pagliaroli, arXiv:0807.1301 [astro-ph], (2008).

[26] Stuart Samuel, Phys. Rev. D 48, 1462 (1993).

[27] B. Dasgupta, A. Mirizzi, I. Tamborra, and R. Tomàs, Phys. Rev. D 81, 093008 (2010).

[28] J. Pantaleone, Phys. Lett. B 287, 128 (1992).

[29] J. Pantaleone, Phys. Rev. D 46, 510 (1992).

[30] G. Sigl and G. Raffelt, Nucl. Phys. B 406, 423 (1993).

[31] T. Lund and J. P. Kneller, Phys. Rev. D 88023008 (2013).

[32] Y. Pehlivan, A. B. Balantekin, T. Kajino, and T. Yoshida, Phys. Rev. D 84065008 (2011).

[33] Y. Pehlivan, A. B. Balantekin, and T. Kajino, Phys. Rev. D 90065011 (2014).

[34] H. Duan, G. M. Fuller, and Y. Z. Qian, Phys. Rev. D 74, 123004 (2006).

[35] H. Duan, G. M. Fuller, J. Carlson, and Y.-Z. Qian, Phys. Rev. D 74105014 (2006).

[36] B. Dasgupta, and A. S. Dighe, Phys. Rev. D 77, 113002 (2008).

[37] D. Väänänen, C. Volpe, J. Cosm. Astropart. Phys. 10, 019 (2011).

[38] A. Mirizzi, and R. Tomas, Phys. Rev. D 84033013 (2011).

[39] L. Wolfenstein, Phys. Rev. D 17, 2369 (1978).

[40] G. M. Fuller, R. W. Mayle, J. R. Wilson, and D. N. Schramm, Astrophys. J 322, 795 (1987).

[41] A. S. Dighe, and A. Yu. Smirnov, Phys. Rev. D 62, 033007 (2000).

[42] A. Y. Smirnov, arXiv: 0305106v1 [hep-ph] (2003).

[43] H. Duan, G. M. Fuller, and Y.-Z. Qian, Ann. Rev. Nucl. Part. Sci. 60, 569 (2010).

[44] H. Duan and J. P. Kneller, J. Phys. G 36, 113201 (2009).

[45] G. L. Fogli et al., Phys. Rev. D 86, 013012 (2012).

[46] W. C. Haxton, Phys. Rev. D 36, 2283 (1987).

[47] J. Engel, E. Kolbe, K. Langanke, and P. Vogel, Phys. Rev. C 54, 2740 (1996).

[48] A. C. Hayes and I. S. Towner, Phys. Rev. C 61, 044603 (2000).

[49] J. M. Sampaio, K. Langanke, G. Martinez-Pinedo, Phys. Lett. B511, 11 (2001).

[50] T. Suzuki, M. Honma, K. Higashiyama, T. Yoshida, T. Kajino, T. Otsuka, H. Umeda, and K. Nomoto, Phys. Rev. C 79, 061603(R) (2009).

[51] N. Auerbach, N. Van Giai, and O. K. Vorov, Phys. Rev. C 56, R2368 (1997).

[52] S. K. Singh, N. C. Mukhopadhyay, and E. Oset, Phys. Rev. C 57, 2687 (1998).

[53] C. Volpe, N. Auerbach, G. Colò, T. Suzuki, and N. Van Giai, Phys. Rev. C 62, 015501 (2000).

[54] C. Volpe, N. Auerbach, G. Colò, and N. Van Giai, Phys. Rev. C 65, 044603 (2002).

[55] E. Kolbe, K. Langanke, S. Krewald, F.-K. Thielemann, Nucl. Phys. A 540, 599 (1992).

[56] E. Kolbe, K. Langanke, F.-K. Thielemann, and P. Vogel, Phys. Rev. C 52, 3437 (1995).

[57] N. Jachowicz, S. Rombouts, K. Heyde, and J. Ryckebusch, Phys. Rev. C 59, 3246 (1999).

[58] N. Jachowicz, K. Heyde, J. Ryckebusch, and S. Rombouts, Phys. Rev. C 65, 025501 (2002).

[59] A. Botrugno and G. Co', Nucl. Phys. A 761, 200 (2005).

[60] E. Kolbe, K. Langanke, and P. Vogel, Nucl. Phys. A 652, 91 (1999).

[61] E. Kolbe, K. Langanke, G. Martínez-Pinedo, and P. Vogel, J. Phys. G 29, 2569 (2003).

[62] P. Vogel, Nucl. Phys. A 777, 340 (2006).

[63] J. D. Walecka Muon Physics, edited by V. M. Hughes and C. S. Wu, Academic, New York (1975).

[64] T. K. Gaisser and J. S. O’Connell, Phys. Rev. D 34, 822 (1986).

[65] T. Kuramoto, M. Fukugita, Y. Kohyama, and K. Kubodera, Nucl. Phys. A 512, 711 (1990).

[66] R. Lazauskas and C. Volpe, Nucl. Phys. A 792, 219 (2007).

[67] M. K. Cheoun, E. Ha, K. S. Kim, and T. Kajino, J. Phys. G 37, 055101 (2010).

[68] V. C. Chasioti, T. S. Kosmas, Nucl. Phys. A 829, 234 (2009).

[69] V. Tsakstara, T. S. Kosmas, Phys. Rev. C 84, 064620 (2011).

[70] V. Tsakstara, T. S. Kosmas, Phys. Rev. C 86, 044618 (2012).

[71] A. R. Samana, F. Krmpotic, N. Paar, and C. A. Bertulani, Phys. Rev. C 83, 024303 (2011).

[72] N. Paar, D. Vretenar, T. Marketin, P. Ring, Phys. Rev. C 77, 024608 (2008). 
[73] E. Kolbe and K. Langanke, Phys. Rev. C 63, 025802 (2001).

[74] J. Engel, G. C. McLaughlin, C. Volpe, Phys. Rev. D 67, 013005 (2003).

[75] N. Paar, H. Tutman, T. Marketin, and T. Fischer, Phys. Rev. C 87, 025801 (2013).

[76] N. Paar, T. Suzuki, M. Honma, T. Marketin, and D. Vretenar, Phys. Rev. C 84, 047305 (2011).

[77] U. Raha, F. Myhrer, and K. Kubodera, Phys. Rev. C 85, 045502 (2012).

[78] U. Raha, F. Myhrer, and K. Kubodera, Phys. Rev. C 86, 039903(E) (2012).

[79] H.-Th. Janka, K. Langanke, A. Marek, and G. Martinez-Pìnedo, Phys. Rep. 442, 38 (2007).

[80] S. Choubey, B. Dasgupta, A. Dighe, and A. Mirizzi, arXiv: 1008.0308 [hep-ph] (2010).

[81] S. Chakraborty, T. Fischer, A. Mirizzi, N. Saviano, and R. Tomas, Phys. Rev. D 84, 025002, (2011).

[82] S. Chakraborty, A. Mirizzi, N. Saviano, and D. S. Seixas, Phys. Rev. D 89, 093001, (2014).

[83] A. Banerjee, A. Dighe, and G. Raffelt, Phys. Rev. D 84, 053013, (2011).

[84] G. Raffelt, S. Sarikas, and D. S. Seixas, Phys. Rev. Lett. 111, 091101 (2013).

[85] G.G.Raffelt, M.Th.Keil, R.Buras, H.-T.Janka, M.Rampp (MPA, Garching), Proceedings NOON 03, Kanazawa (2003).

[86] M. T. Keil, Supernova neutrino spectra and applications to flavor oscillations, dissertation, Technische Universitaet Muenchen, arXiv:0308228 [astro-ph], (2003).

[87] I. Tamborra, B. Müller, L. Hüdepohl, H.-Th. Janka, and Georg Raffelt, Phys. Rev. D 86, 125031 (2012).

[88] J. S. O' Connel et al., Phys. Rev. C 6, 719 (1972).

[89] G. A. Lalazissis, T. Nikšić, D. Vretenar, and P. Ring, Phys. Rev. C 71, 024312 (2005).

[90] J. F. Berger, M. Girod, and D. Gogny, Comp. Phys. Comm. 63, 365 (1991).

[91] Y. F. Niu, N. Paar, D. Vretenar, and J. Meng, Phys. Rev. C 83, 045807 (2011).

[92] W. Hauser and H. Feshbach, Phys. Rev. 87, 366 (1952).

[93] T. Rauscher, code SMARAGD, v0.9.3s (2014).

[94] T. Rauscher, Int. J. Mod. Phys. E 20, 1071 (2011).

[95] T. Rauscher and F. K. Thielemann, At. Data Nucl. Data Tables 75, 1 (2000).

[96] National Nuclear Data Center, NuDat 2 database, http://www.nndc.bnl.gov/nudat2/

[97] D. Mocelj, T. Rauscher, G. Martínez-Pinedo, K. Langanke, L. Pacearescu, A. Fäßler, F.-K. Thielemann, and Y. Alhassid, Phys. Rev. C 75, 045805 (2007).

[98] G. Audi, A. H. Wapstra, and C. Thibault, Nucl. Phys. A729, 337 (2003).

[99] K. Scholberg, private communications (2015).

[100] D. V. Forero, M. Tórtola, and J. W. F. Valle, Phys. Rev. D 86 073012, (2012).

[101] M. C. Gonzales-Garcia, M. Maltoni, J. Salvado, and T. Schwetz, J. High. En. Phys. 12, 123 (2012).

[102] K. Scholberg, arXiv:1205.6003 [hep-ph] (2012).

[103] G. M. Fuller, W. C. Haxton, and G. C. McLaughlin, Phys. Rev. D 59, 085005, (1999).

[104] A. Bolozdynya et al, arXiv: 1211.5199 [hep-ex] (2012).

[105] B. B. Kinsey, G. A. Bartholomew, and W. H. Walker, Phys. Rev. 82, 3 (1951).

[106] A. Burrows, D. Klein, and R. Gandhi, Phys. Rev. D 45, 3361 (1992).

[107] M. Apollonio et al, Eur. Phys. J. C 27, 331 (2003).

[108] K. Abe et al., Nucl. Instr. Meth. A 737, 253 (2014). 
TABLE I: Initial lumininosity ratios of (anti)neutrino species for type II supernova spectra in accretion(A) and cooling(C) phase. Three possible combinations of the luminosity ratios for (anti)neutrino species are shown for each phase, together with fixed total energy emitted by (anti)neutrinos and time duration of the phases.

\begin{tabular}{|c|c|c|}
\hline & $l_{\nu_{\mathrm{e}}}: l_{\bar{\nu}_{\mathrm{e}}}: l_{\nu_{x}}$ & $T_{i n t}$ \\
\hline Accretion ph. & $\approx 0.4 \times 10^{53} \mathrm{erg}$ & \\
\hline $\mathrm{A} 1$ & $4.0: 4.0: 3.0$ & $0.4 \mathrm{~s}$ \\
\hline A 2 & $3.0: 3.0: 1.0$ & \\
\hline A 3 & $12.0: 8.0: 5.0$ & \\
\hline Cooling ph. & $\approx 2 \times 10^{53}$ erg & \\
\hline $\mathrm{C} 1$ & $1.0: 1.0: 1.0$ & $10.0 \mathrm{~s}$ \\
\hline $\mathrm{C} 2$ & $2.0: 2.0: 4.0$ & \\
\hline
\end{tabular}

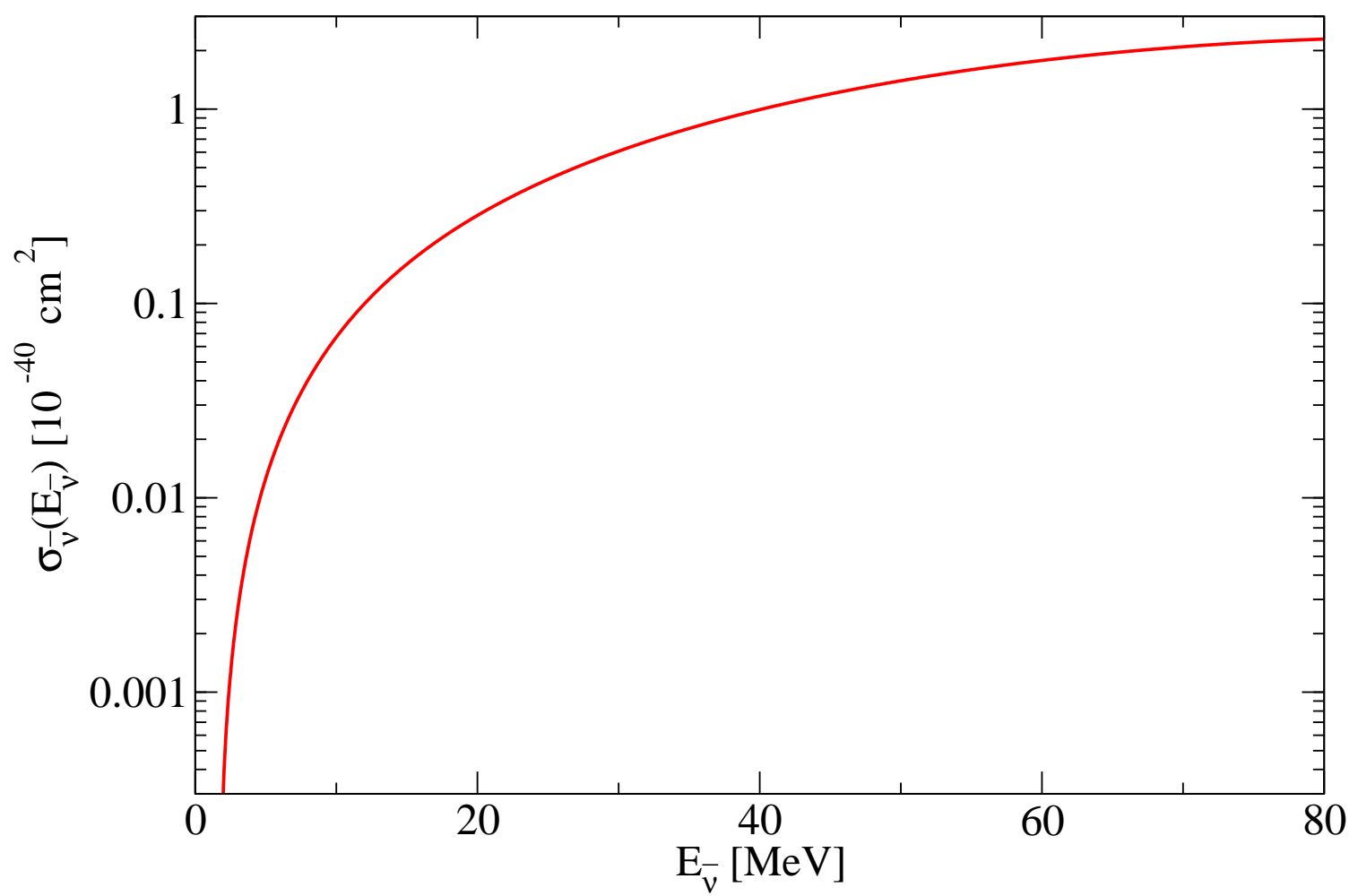

FIG. 1: The cross sections for the inelastic scattering of $\bar{\nu}_{e}$ on free protons, shown as a function of the incoming antineutrino energy. Calculations are based on heavy-baryon chiral perturbation theory with radiative corrections from Ref. [77, 78]. 
TABLE II: Detector response for $\nu_{\mathrm{e}}\left(\bar{\nu}_{\mathrm{e}}\right)$-induced reactions in mineral oil $\left(\mathrm{CH}_{2}\right)$, water $\left(\mathrm{H}_{2} \mathrm{O}\right),{ }^{56} \mathrm{Fe}$ and ${ }^{208} \mathrm{~Pb}$, for the incoming (anti)neutrino fluxes of the accretion supernova phase, both for normal (NH) and inverted (IH) neutrino mass hierarchies. Only dominant emission channels are shown, including $\mathrm{e}^{+}$, one- and two-neutron emissions. A1, A2, A3 denote the combinations of the luminosity ratios of (anti)neutrino species given in Tab. I while (i) stands for $\left\langle E_{\nu_{\mathrm{e}}}^{0}\right\rangle=8 \mathrm{MeV},\left\langle E_{\bar{\nu}_{\mathrm{e}}}^{0}\right\rangle=11 \mathrm{MeV},\left\langle E_{\nu_{x}}^{0}\right\rangle=$ $13 \mathrm{MeV}$; (ii) is $\left\langle E_{\nu_{\mathrm{e}}}^{0}\right\rangle=10 \mathrm{MeV},\left\langle E_{\bar{\nu}_{\mathrm{e}}}^{0}\right\rangle=13 \mathrm{MeV},\left\langle E_{\nu_{x}}^{0}\right\rangle=15 \mathrm{MeV}$; and (iii) is $\left\langle E_{\nu_{\mathrm{e}}}^{0}\right\rangle=12 \mathrm{MeV},\left\langle E_{\bar{\nu}_{\mathrm{e}}}^{0}\right\rangle=15 \mathrm{MeV},\left\langle E_{\nu_{x}}^{0}\right\rangle=$ $19 \mathrm{MeV}$.

\begin{tabular}{|c|c|c|c|c|c|c|c|}
\hline \multirow{2}{*}{$\begin{array}{l}\text { Accretion ph. } \\
0.4 \mathrm{~s}\end{array}$} & \multirow[t]{2}{*}{ L type } & \multicolumn{2}{|c|}{ (i) } & \multicolumn{2}{|c|}{ (ii) } & \multicolumn{2}{|c|}{ (iii) } \\
\hline & & $\mathrm{NH}$ & $\mathrm{IH}$ & $\mathrm{NH}$ & $\mathrm{IH}$ & $\mathrm{NH}$ & $\mathrm{IH}$ \\
\hline \multicolumn{8}{|c|}{$\mathrm{p}\left(\bar{\nu}_{\mathrm{e}}, \mathrm{e}^{+}\right) \mathrm{n}$ in $\mathrm{CH}_{2}$} \\
\hline & A1 & $45-60$ & $42-56$ & $54-70$ & $49-64$ & $65-82$ & $61-78$ \\
\hline & A 2 & $58-74$ & $28-39$ & $69-87$ & $32-45$ & $82-101$ & $41-55$ \\
\hline & A3 & $43-57$ & $34-47$ & $51-67$ & $40-54$ & $61-78$ & $51-66$ \\
\hline \multicolumn{8}{|c|}{$\mathrm{p}\left(\bar{\nu}_{\mathrm{e}}, \mathrm{e}^{+}\right) \mathrm{n}$ in $\mathrm{H}_{2} \mathrm{O}$} \\
\hline & A1 & $34-47$ & $32-44$ & $41-55$ & $37-50$ & $49-65$ & $47-62$ \\
\hline & $\mathrm{A} 2$ & $44-58$ & $21-31$ & $53-69$ & $25-36$ & $62-79$ & $31-43$ \\
\hline & A3 & $33-45$ & $26-37$ & $39-53$ & $30-43$ & $47-61$ & $39-52$ \\
\hline \multicolumn{8}{|c|}{${ }^{56} \mathrm{Fe}\left(\nu_{\mathrm{e}}, \mathrm{e}^{-}\right){ }^{56} \mathrm{Co}$} \\
\hline \multirow[t]{3}{*}{$\ln$} & $\mathrm{A} 1$ & $0-4$ & $0-3$ & $2-6$ & $2-6$ & $6-13$ & $6-13$ \\
\hline & $\mathrm{A} 2$ & $0-2$ & $0-2$ & $1-4$ & $1-4$ & $4-9$ & $4-9$ \\
\hline & A3 & $0-3$ & $0-3$ & $1-5$ & $1-5$ & $5-11$ & $5-11$ \\
\hline \multirow[t]{3}{*}{ 1n } & A1 & $12-20$ & $12-20$ & $19-29$ & $19-29$ & $35-48$ & $35-48$ \\
\hline & $\mathrm{A} 2$ & $7-14$ & $7-14$ & $12-20$ & $12-20$ & $22-33$ & $22-33$ \\
\hline & A3 & $10-17$ & $10-17$ & $16-25$ & $16-25$ & $29-41$ & $29-41$ \\
\hline \multirow[t]{3}{*}{$2 \mathrm{n}$} & A1 & $0-3$ & $0-3$ & $1-5$ & $1-5$ & $7-14$ & $7-14$ \\
\hline & $\mathrm{A} 2$ & $0-2$ & $0-2$ & $0-4$ & $0-4$ & $4-10$ & $4-10$ \\
\hline & A3 & $0-2$ & $0-2$ & $1-5$ & $1-5$ & $6-12$ & $6-12$ \\
\hline \multirow[t]{3}{*}{ total n } & A1 & $15-24$ & $15-24$ & $26-38$ & $26-38$ & $56-72$ & $56-72$ \\
\hline & $\mathrm{A} 2$ & $9-17$ & $9-17$ & $17-26$ & $17-26$ & $36-49$ & $36-49$ \\
\hline & A3 & $12-20$ & $12-20$ & $22-32$ & $22-32$ & $46-61$ & $46-61$ \\
\hline
\end{tabular}


TABLE III: The same as Tab. II but for the cooling supernova phase. C1, C2, C3 denote the combinations of the luminosity ratios for (anti)neutrino species given in Tab. [

\begin{tabular}{|c|c|c|c|c|c|c|c|}
\hline \multirow{2}{*}{$\begin{array}{l}\text { Cooling ph. } \\
10 \mathrm{~s}\end{array}$} & \multirow[t]{2}{*}{ L type } & \multicolumn{2}{|c|}{ (i) } & \multicolumn{2}{|c|}{ (ii) } & \multicolumn{2}{|c|}{ (iii) } \\
\hline & & $\mathrm{NH}$ & $\mathrm{IH}$ & $\mathrm{NH}$ & $\mathrm{IH}$ & $\mathrm{NH}$ & $\mathrm{IH}$ \\
\hline \multicolumn{8}{|c|}{$\mathrm{p}\left(\bar{\nu}_{\mathrm{e}}, \mathrm{e}^{+}\right) \mathrm{n}$ in $\mathrm{CH}_{2}$} \\
\hline & $\mathrm{C} 1$ & $253-285$ & $248-281$ & $303-339$ & $295-331$ & $394-435$ & $370-409$ \\
\hline & $\mathrm{C} 2$ & $217-248$ & $211-242$ & $270-304$ & $257-291$ & $378-418$ & $340-378$ \\
\hline & C3 & $235-267$ & $230-262$ & $286-320$ & $276-310$ & $381-421$ & $352-391$ \\
\hline \multicolumn{8}{|c|}{$\mathrm{p}\left(\bar{\nu}_{\mathrm{e}}, \mathrm{e}^{+}\right) \mathrm{n}$ in $\mathrm{H}_{2} \mathrm{O}$} \\
\hline & $\mathrm{C} 1$ & $195-224$ & $192-220$ & $234-266$ & $228-259$ & $305-341$ & $286-321$ \\
\hline & $\mathrm{C} 2$ & $167-194$ & $163-190$ & $209-238$ & $199-228$ & $292-327$ & $263-296$ \\
\hline & C3 & $181-209$ & $178-205$ & $220-251$ & $213-243$ & $294-330$ & $272-306$ \\
\hline \multicolumn{8}{|c|}{${ }^{56} \mathrm{Fe}\left(\nu_{\mathrm{e}}, \mathrm{e}^{-}\right){ }^{56} \mathrm{Co}$} \\
\hline \multirow[t]{3}{*}{ 1n } & $\mathrm{C} 1$ & $11-19$ & $7-14$ & $21-31$ & $13-21$ & $46-60$ & $27-39$ \\
\hline & $\mathrm{C} 2$ & $14-23$ & $9-16$ & $25-36$ & $15-24$ & $55-71$ & $30-42$ \\
\hline & C3 & $12-20$ & $7-14$ & $22-32$ & $14-22$ & $48-63$ & $28-40$ \\
\hline \multicolumn{8}{|c|}{${ }^{208} \mathrm{~Pb}\left(\nu_{\mathrm{e}}, \mathrm{e}^{-}\right)^{208} \mathrm{Bi}$} \\
\hline \multirow[t]{3}{*}{$\ln$} & $\mathrm{C} 1$ & $88-108$ & $68-85$ & $126-150$ & $95-116$ & $194-223$ & $139-163$ \\
\hline & $\mathrm{C} 2$ & $106-128$ & $82-101$ & $151-177$ & $111-133$ & $230-262$ & $154-180$ \\
\hline & C3 & $93-113$ & $71-89$ & $133-157$ & $99-121$ & $204-234$ & $144-169$ \\
\hline \multirow[t]{3}{*}{$2 \mathrm{n}$} & $\mathrm{C} 1$ & $9-16$ & $4-9$ & $19-29$ & $10-17$ & $53-69$ & $28-40$ \\
\hline & $\mathrm{C} 2$ & $11-18$ & $5-11$ & $23-34$ & $11-19$ & $63-80$ & $31-44$ \\
\hline & C3 & $9-16$ & $4-10$ & $20-30$ & $10-18$ & $56-72$ & $29-41$ \\
\hline \multirow[t]{3}{*}{ total $\mathrm{n}$} & $\mathrm{C} 1$ & $112-134$ & $82-101$ & $173-201$ & $122-145$ & $313-350$ & $205-235$ \\
\hline & $\mathrm{C} 2$ & $135-159$ & $98-119$ & $207-237$ & $141-166$ & $371-410$ & $228-259$ \\
\hline & C3 & $118-141$ & $86-105$ & $182-210$ & $128-151$ & $329-366$ & $213-243$ \\
\hline
\end{tabular}




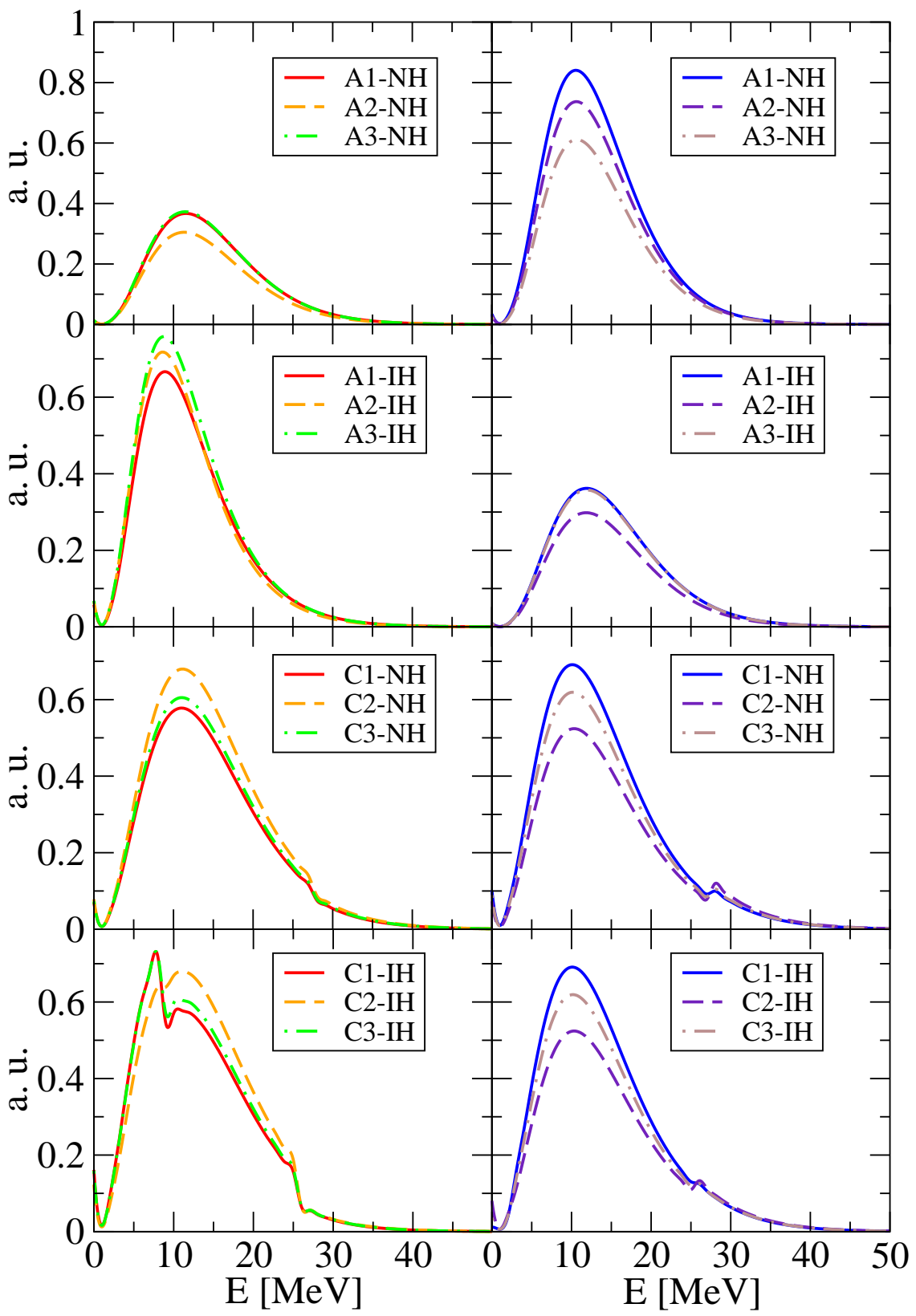

FIG. 2: Incoming $\nu_{\mathrm{e}}$ (left side) and $\bar{\nu}_{\mathrm{e}}$ fluxes (right side) for the accretion (A) and cooling (C) phase of core-collapse supernovae as a function of neutrino energy for normal $(\mathrm{NH})$ and inverted neutrino mass hierarchy (IH), for initial $\nu$ energy configuration (ii). Three combinations of the luminosity ratios for (anti)neutrino species used in the accretion (A1-3) and cooling (C1-3) phases are given in Tab. I. 


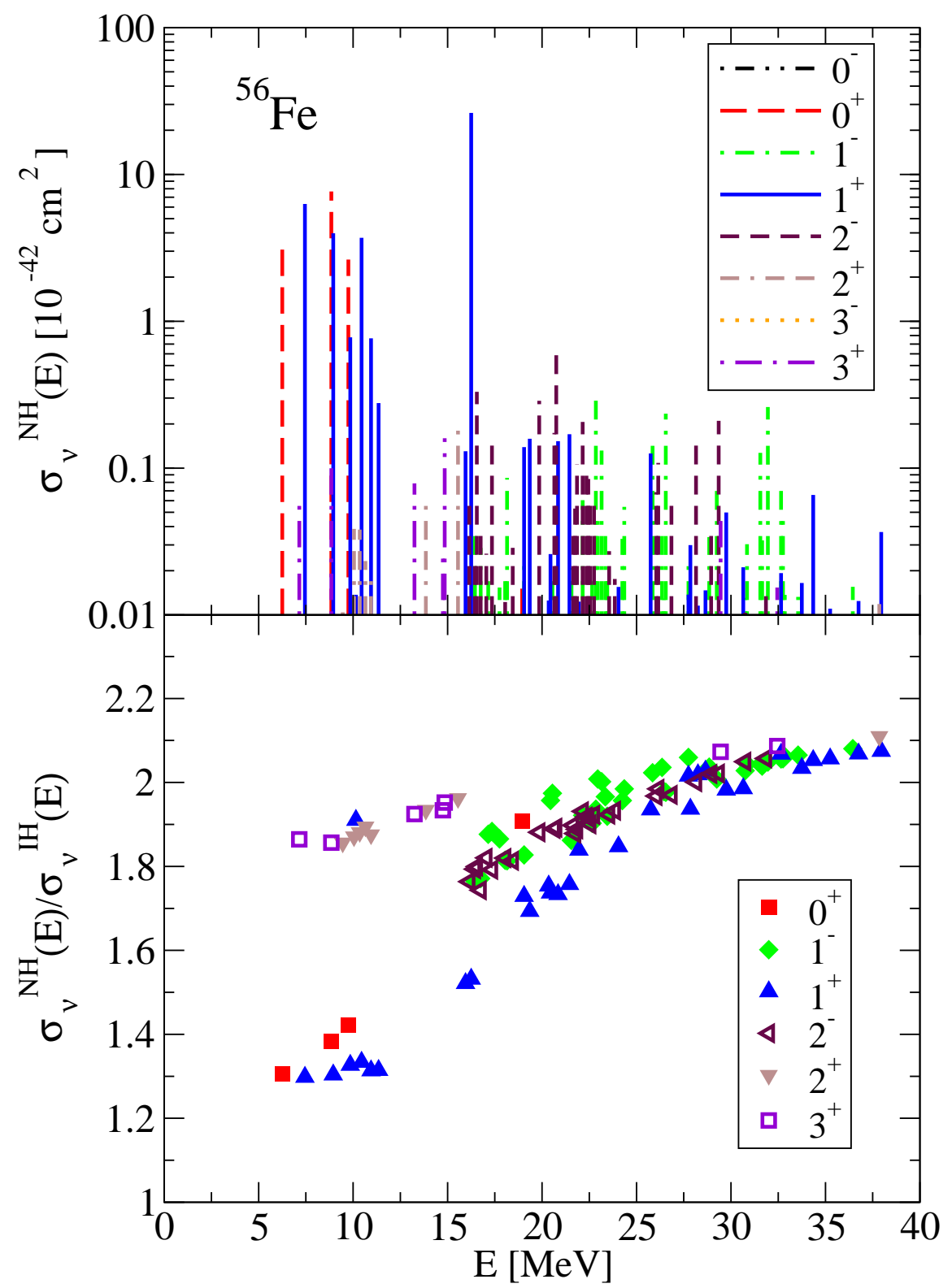

FIG. 3: Multipole decomposition of the flux averaged cross sections of the $\nu_{\mathrm{e}^{-}}{ }^{56} \mathrm{Fe}$ reaction as a function of excitation energy in the initial nucleus, calculated for the incoming cooling phase neutrino spectra (C3) in normal hierarchy (NH), including multipoles $J^{\pi}=0^{ \pm}-3^{ \pm}$(upper panel). The ratio of cross sections between the normal (NH) and inverted (IH) neutrino mass hierarchies is shown in the lower panel. 


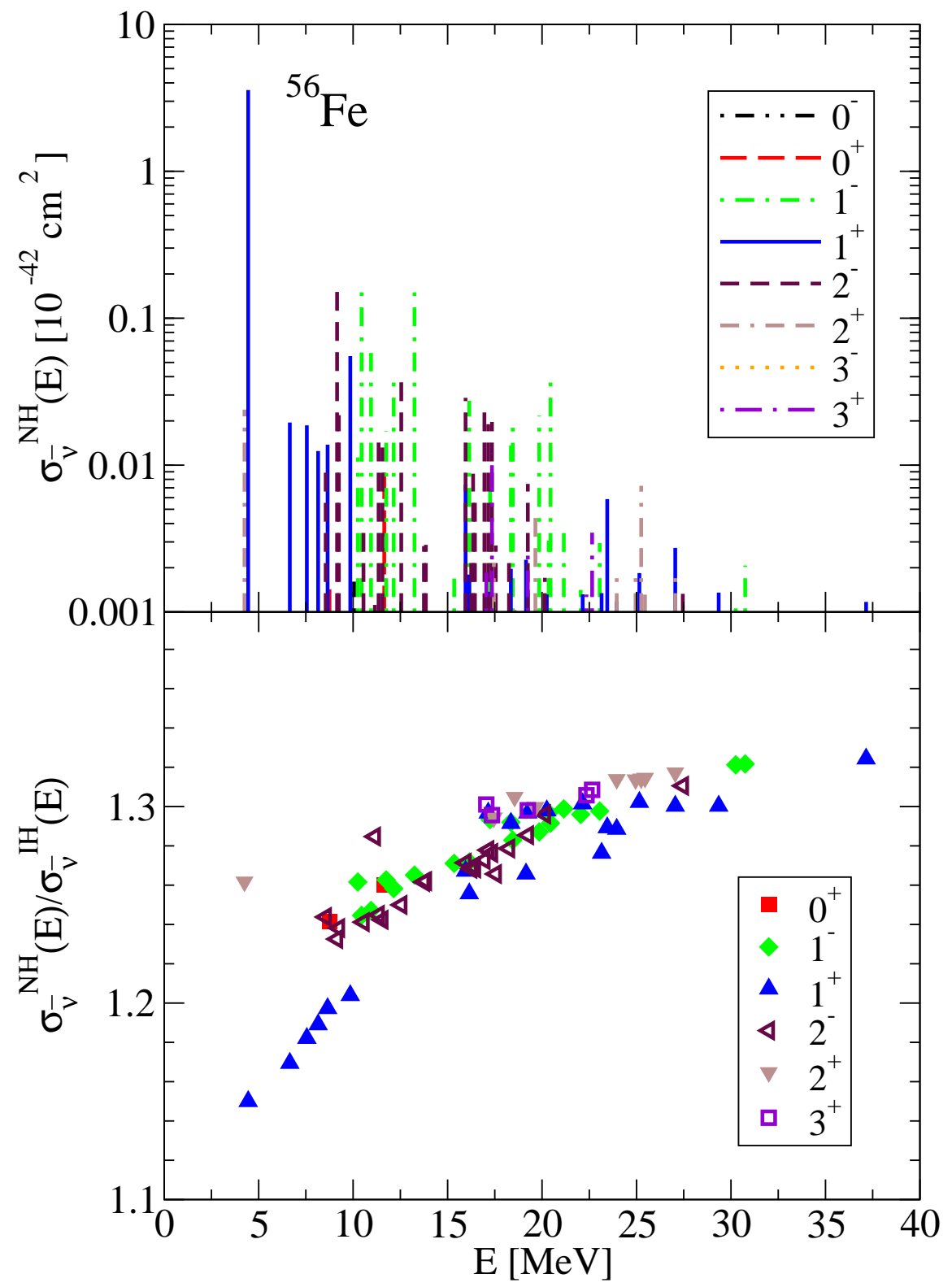

FIG. 4: Same as Fig. 3 but for the $\bar{\nu}_{\mathrm{e}^{-}}{ }^{56}$ Fe reaction. 


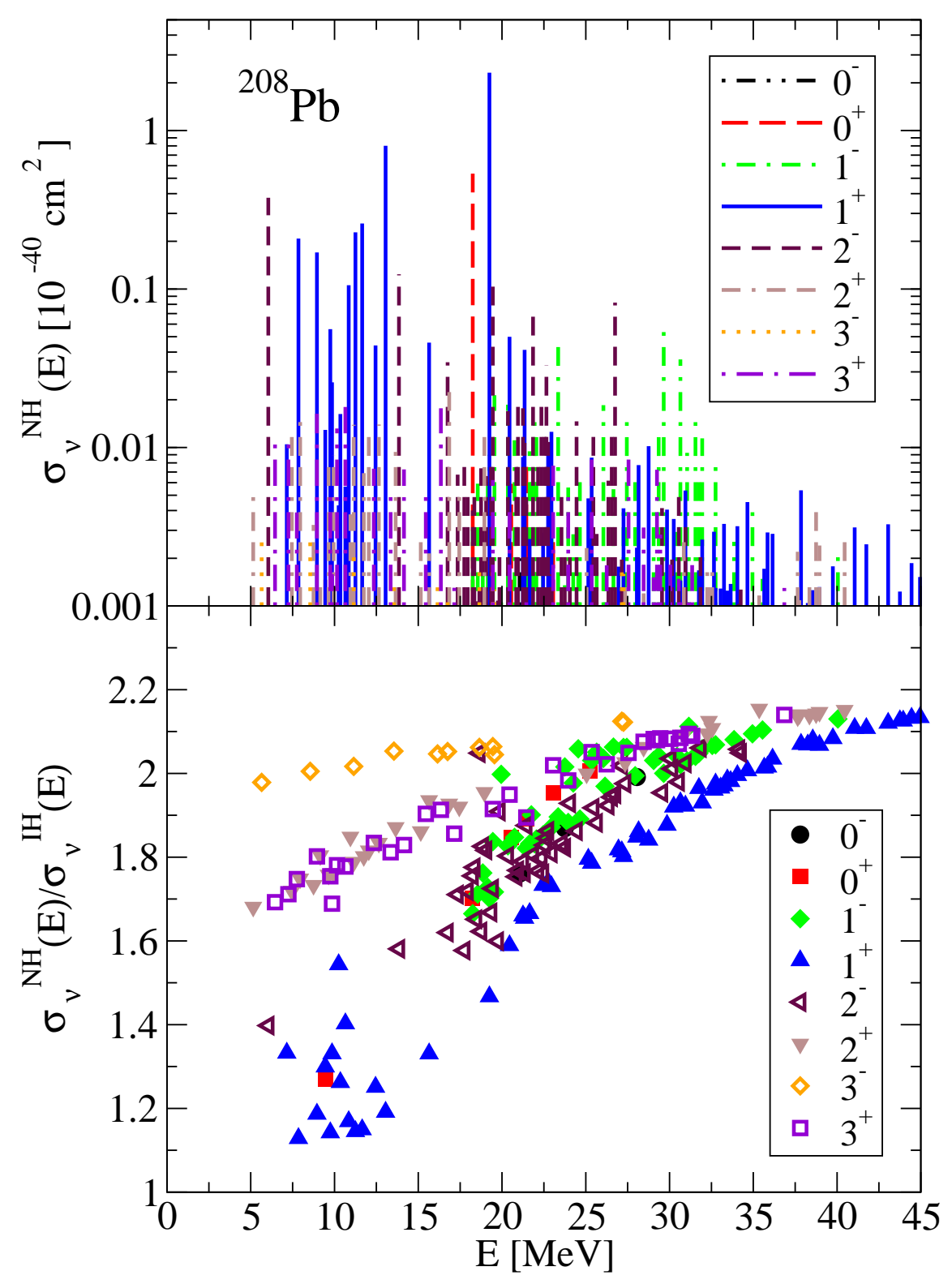

FIG. 5: Same as Fig. 3 but for the $\nu_{\mathrm{e}^{-}}{ }^{208} \mathrm{~Pb}$ reaction. 
(i)

(ii)

(iii)

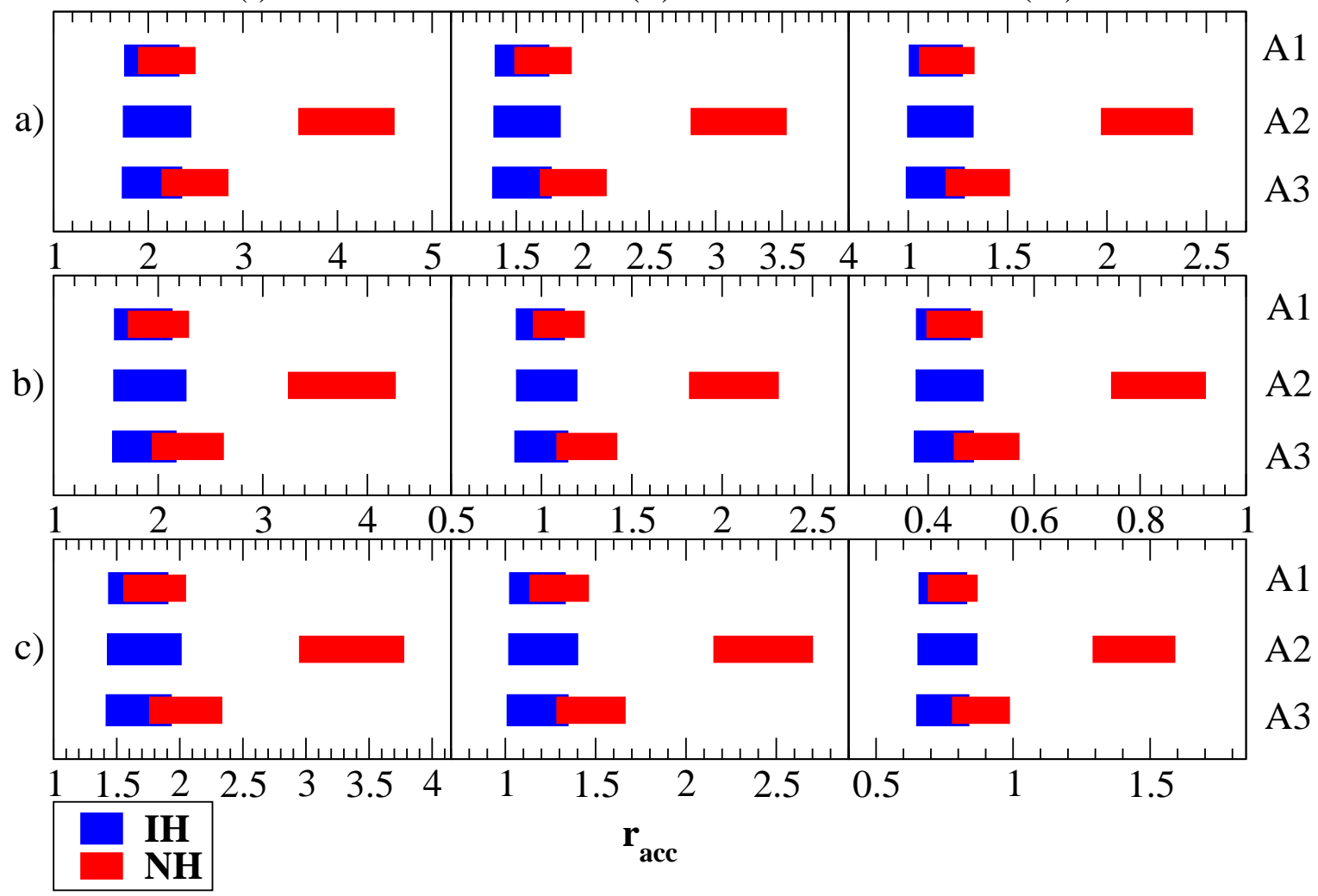

FIG. 6: The ratios $r_{a c c}$ of the number of the detector events induced in mineral oil $\left(\mathrm{CH}_{2}\right)$, water $\left(\mathrm{H}_{2} \mathrm{O}\right)$, and ${ }^{208} \mathrm{~Pb}$ for the incoming (anti)neutrino fluxes of the accretion phase in normal $(\mathrm{NH})$ and inverted $(\mathrm{IH})$ mass hierarchy: (a) $r_{\mathrm{e}}^{\text {free }, 1 \mathrm{p}, \mathrm{Pb}}\left[10^{-1}\right]$ (b) $r_{\mathrm{e}^{+}, 2 \mathrm{p}}^{\text {free } \mathrm{Pb}}$ (c) $r_{\mathrm{e}^{+}, \text {tot n }}^{\text {free }, \mathrm{Pb}}\left[10^{-1}\right]$. The three columns correspond to (i) $\left\langle E_{\nu_{\mathrm{e}}}^{0}\right\rangle=8 \mathrm{MeV},\left\langle E_{\bar{\nu}_{\mathrm{e}}}^{0}\right\rangle=11 \mathrm{MeV},\left\langle E_{\nu_{x}}^{0}\right\rangle=13 \mathrm{MeV} ;(\mathrm{ii})$ $\left\langle E_{\nu_{\mathrm{e}}}^{0}\right\rangle=10 \mathrm{MeV},\left\langle E_{\bar{\nu}_{\mathrm{e}}}^{0}\right\rangle=13 \mathrm{MeV},\left\langle E_{\nu_{x}}^{0}\right\rangle=15 \mathrm{MeV}$; and (iii) $\left\langle E_{\nu_{\mathrm{e}}}^{0}\right\rangle=12 \mathrm{MeV},\left\langle E_{\bar{\nu}_{\mathrm{e}}}^{0}\right\rangle=15 \mathrm{MeV},\left\langle E_{\nu_{x}}^{0}\right\rangle=19 \mathrm{MeV} . \mathrm{A} 1, \mathrm{~A} 2$, A3 denote the combinations of the luminosity ratios for the accretion phase given in Tab. [] 
(i)

(ii)

(iii)

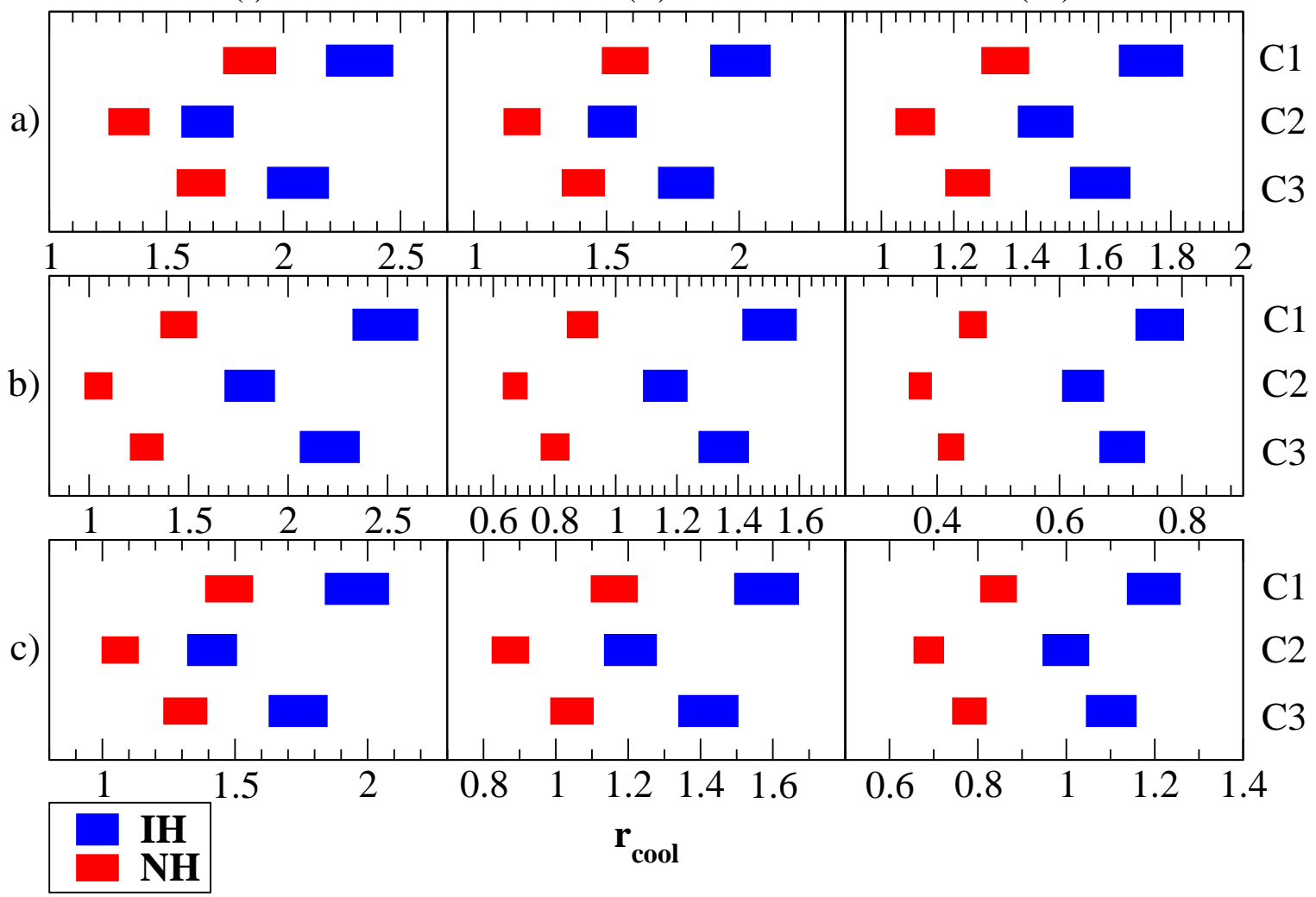

FIG. 7: The ratios $r_{\text {cool }}$ of the number of the detector events induced in mineral oil $\left(\mathrm{CH}_{2}\right)$, water $\left(\mathrm{H}_{2} \mathrm{O}\right)$, and ${ }^{208} \mathrm{~Pb}$ for the incoming (anti)neutrino fluxes of the cooling phase in normal (NH) and inverted (IH) mass hierarchy for three configurations of initial $\nu$ average energies. The same notation as in Fig. 6] applies. C1, C2, C3 denote the combinations of the luminosity ratios for the cooling phase given in Tab. [ 